\title{
Robert GUELLUY
}

Chanoine, Maître en théologie, Grand Séminaire de Turnai (Belgique), professeur ordinaire, Université Catholique de Louvain

(2003)

\section{Robert Guelluy}

\section{Sa vie, sa pensée, son œuvre.}

Textes recueillis et présentés par Sully Faïk.

\author{
Un document produit en version numérique par Sully Faïk, \\ Docteur en philosophie et en lettres \\ professeur retraité de l'Université Lovanium de Kinshasa (RDC) \\ et de l'Université de Niamey (Niger). \\ Courriel:nzuji@acla.ucl.ac.be
}

Dans le cadre de la collection: "Les classiques des sciences sociales"

Site web: http://classiques.uqac.ca/

Une collection développée en collaboration avec la Bibliothèque

Paul-Émile-Boulet de l'Université du Québec à Chicoutimi

Site web: http://bibliotheque.uqac.ca/ 
Cette édition électronique a été réalisée par Sully Faïk, bénévole, Docteur en philosophie et en lettres, professeur retraité de l'Université Lovanium de Kinshasa (RDC) et de l'Université de Niamey (Niger).

à partir de :

Robert Guelluy. Sa vie, sa pensée, son œuvre. Textes recueillis et présentés par Sully Faïk.

Louvain-la-Neuve, 2003, 127 pp.

M Sully Fal̈k, Docteur en philosophie et en lettres, professeur retraité de l'Université Lovanium de Kinshasa (RDC) et de l'Université de Niamey (Niger), nous a accordé le 26 juin 2004 son autorisation de diffuser électroniquement ce livre.

Documents inédits et libres de droits. Textes publiés avec l'autorisation de MM. Robert Guelluy et Sully Faïk.

Courriel :nzuji@acla.ucl.ac.be

Polices de caractères utilisée :

Pour le texte: Times New Roman, 14 points.

Pour les citations : Times New Roman, 12 points.

Pour les notes de bas de page : Times New Roman, 12 points.

Édition électronique réalisée avec le traitement de textes Microsoft Word 2004 pour Macintosh.

Mise en page sur papier format : LETTRE (US letter), 8.5” x 11”)

Édition numérique réalisée le 22 novembre 2006 à Chicoutimi, Ville de Saguenay, province de Québec, Canada. 
Texte publié avec les encouragements de Monsieur l'abbé Minet, actuel Président du grand Séminaire de Tournai, et de Monseigneur Harpigny, Évêque du diocèse de Tournai

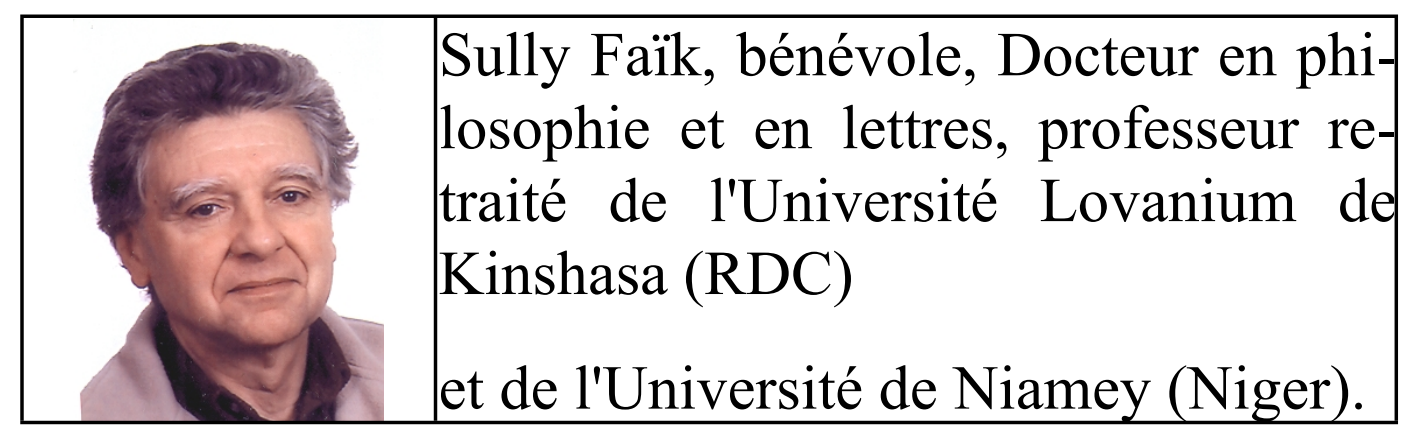




\section{Robert Guelluy (2003)}

\section{Robert Guelluy. Sa vie, sa pensée, son œuvre. Textes recueillis et présentés par Sully Faïk.}

\section{Robert Guelluy}

Sa vie, sa pensée, son auvre

\section{Mots à vivre}

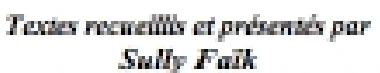

Louvain-Ia-Neuve 2003

Louvain-la-Neuve, 2003, 127 pp. 


\section{Table des matières}

Introduction, par Sully Faïk

Robert Guelluy, sa vie.

$\underline{\text { Robert Guelly, sa pensée }}$

Mots à vivre

$$
\begin{aligned}
& \underline{\mathrm{A}}-\underline{\mathrm{B}}-\underline{\mathrm{C}}-\underline{\mathrm{D}}-\underline{\mathrm{E}}-\underline{\mathrm{F}}-\underline{\mathrm{G}}-\underline{\mathrm{H}}-\underline{\mathrm{I}}-\underline{\mathrm{J}}-\underline{\mathrm{L}}-\underline{\mathrm{M}}-\underline{\mathrm{O}}-\underline{\mathrm{P}}-\underline{\mathrm{Q}}-\underline{\mathrm{R}} \\
& -\underline{\mathrm{S}}-\underline{\mathrm{T}}-\underline{\mathrm{U}}-\underline{\mathrm{V}} .
\end{aligned}
$$

Bibliographie 


\title{
Robert Guelluy
}

\section{Sa vie, sa pensée, son ouvre}

\author{
Mots à vivre
}

\section{Textes recueillis et présentés par Sully Faïk}

\section{Louvain-la-Neuve, 2003}




\section{Introduction}

\section{$\underline{\text { Retour à la table des matières }}$}

Nombreuses sont les demeures dans la maison de Dieu. Chacun y a la sienne, à nulle autre pareille. C'est comme nos visages : pas deux qui soient identiques! Les chemins de spiritualité, s'ils sont aussi une multitude, se regroupent cependant en de grandes familles, au sein desquelles on vit une plus pure harmonie.

J'avais dix-sept ans, lorsque je découvris l'enseignement du chanoine Guelluy, à l'École des sciences philosophiques et religieuses des Facultés universitaires Saint-Louis à Bruxelles. D'emblée je sentis que je vibrais au même diapason que lui. Sa parole était vraiment celle de mon Dieu. Je ne le quitterai plus. Pas un de ses livres que je ne dévorai dès sa parution.

De tous les maitres spirituels que je rencontrai par la suite, de tous les auteurs que je lus, aucun ne me correspondît jamais avec tant de justesse. Aussi quelle ne fut pas ma joie, lorsque venant m'installer à Louvain-la-Neuve avec ma famille, après une carrière de vingt-deux ans en Afrique, je retrouvai Robert Guelluy et ses homélies à la chapelle de La Source et à l'église paroissiale Saint-François. 
Le projet germa alors de publier les pensées de Robert Guelluy, qu'au cours des années j'avais recueillies dans ses écrits. Le volume fut prêt à la Pentecôte 1991 et le Ministre d'État, Pierre Harmel, en rédigea la préface. Malheureusement, il resta inédit. Une partie de ces textes choisis paraîtront cependant, en 1997, sous le titre Au Gré des pages, dans la brochure Faire fleurir l'instant, dernière publication de Robert Guelluy.

En septembre 1999, je proposai à Robert Guelluy d'enregistrer ses souvenirs. Comme il n'avait plus l'énergie suffisante pour encore écrire, mais qu'il était toujours avide de transmettre son message de vie, il accepta volontiers l'exercice. N'eût été la thrombose qui le terrassa en octobre de cette année-là, nous aurions pu poursuivre les entretiens pendant plusieurs mois.

C'est à partir de ces quelques échanges, trop tôt interrompus, que j'ai rédigé sa biographie. Elle est rigoureusement fidèle à ce qu'il m'a confié. Je me suis seulement permis de réorganiser les informations et d'en uniformiser le style.

J'avais poursuivi aussi le recueil de ses pensées. Parmi les 675 collationnées à ce jour, j'ai repris ici les plus significatives, classées par ordre alphabétique, sous le titre Mots à vivre. Ces phrases brèves et concises, frappées comme des médailles, illustrent un étonnant paradoxe : c'est lorsqu'elles sont exprimées avec le plus de simplicité qu'elles atteignent leur maximum de profondeur et de puissance, qui 
ouvre au voyage intérieur. De la limpidité même des mots et des images naît une profusion de sens si pénétrante qu'elle invite à la méditation. C'est quand en lui le théologien s'efface, pour laisser toute la place à l'homme et à son expérience de vie, que Robert Guelluy devient vraiment diseur de sagesse, diseur de Dieu.

Le volume s'achève sur une bibliographie, que j'ai voulue la plus complète possible. À ma connaissance, elle n'existe nulle part. J'ai tenu à l'établir et à la publier pour rendre l'hommage qu'il mérite à cet être d'exception.

Sully Faïk, 2003. 


\section{Robert Guelluy Sa vie}

Nous ne pouvons nous résigner [...] aux défauts qui défigurent le visage de l'Église, qui empêchent par notre faute d'y reconnaître la face du Christ [...]

Le travail dans la vie du chrétien, p. 147.

\section{Je suis Robert Guelluy}

$\underline{\text { Retour à la table des matières }}$

En janvier 1982, Philippe Mawet interviewait Robert Guelluy, à l'occasion de ses quarante-cinq ans de prêtrise, pour l'émission Radio Louvain-la-Neuve "Du Canal à la Source ». Il commença par la question classique : Monsieur le chanoine pourriez-vous nous dire qui vous êtes? 
Ceux qui ont connu Robert peuvent deviner avec quel sourire amusé mi-railleur, il dut répondre : " Mais Robert Guelluy c'est d'abord Robert Guelluy. Et je tiens beaucoup à être tel ! [...] Je ne désire pas être réduit à une abstraction. Je suis Robert Guelluy. Et ça m'amuse parfois quand je vois les yeux ronds de mes étudiants lorsqu'en rue les gamins crient autour de moi « Robert! Robert !» de toutes leurs forces. C'est ma petite clientèle pour la distribution de bonbons. D'ailleurs elle diminue un peu d'année en année parce qu'elle prend de l'âge et a moins d'intérêt pour ce que je peux lui offrir... »

\section{Naissance}

Robert, Jean, Léon Guelluy est né le 29 juin 1913, vers 6 heures du soir, à Saint-Sauveur, petit village belge du Tournaisis au sud de Renaix. À deux pas de la frontière linguistique et tout près de la France : Lille est à quelques kilomètres. À proximité aussi de Tournai, ville à laquelle il était si attaché. C'est là qu'il suivit sa formation en théologie et où, plus tard, il enseigna. Il aimait répéter, avec son humour habituel, que le clergé belge est le meilleur du monde, celui de Tournai le meilleur de Belgique et celui du Séminaire l'élite du diocèse de Tournai !

Son heure de naissance, ce chanoine pas toujours très canonique, alla s'en enquérir auprès de sa commune d'origine lorsque quel- 
qu'un la lui demanda pour faire son horoscope. Que lui révéla cet horoscope ? Quelle foi y prêta-t-il ? On ne le saura jamais. Mais qu'il se soit livré à cet exercice et qu'il n'hésite pas à en parler est révélateur d'un aspect de son caractère particulièrement curieux mais aussi quelque peu frondeur.

Le nom Guelluy est très localisé dans quelques villages du Tournaisis et du nord de la France. Il est peu fréquent en Belgique. Dans la même région, on trouve des Gueluy (avec un seul L) et, à Lille, des Guilluy : l'un notamment était professeur aux Facultés de Lille et l'autre moine à l'abbaye de la Pierre-qui-Vire. C'étaient des frères, tous deux bénédictins. Ce nom est probablement d'origine écossaise. Une famille noble de Galway, village d'Écosse, portait le nom de « Okelly ». Sur le continent « kelly » aurait donné Guelluy.

\section{Son village natal et ses premières années}

Robert gardait bien des souvenirs de ses premières années et de son village natal. L'image qu'il a de son enfance est extraordinaire. Ses parents étaient de petits agriculteurs. Il menait avec eux une vie très familiale. Ce monde rural paisible, encore médiéval, le fascinait. Il n'y avait qu'un seul téléphone à Saint-Sauveur ! C'était chez le curé. Son oncle a été l'un des premiers, avec le médecin, à rouler en voiture. Tout le monde se connaissait. Tout le monde s'appelait par son

prénom. Les noms de famille n'étaient quasiment pas utilisés. Être 
unis dans le même travail, vivre du même effort, c'est un privilège de la petite entreprise campagnarde, où prédominent les contacts de personne à personne. Les tâches étaient bien réparties. Les hommes coupaient le blé. Les femmes le liaient en bottes. Et les enfants glanaient. Plus tard, le passage à l'agriculture mécanique contribuera à rompre cette belle unité familiale.

\section{Sa famille}

Qui l'a le plus marqué ? Son père ou sa mère ? C'est difficile à dire. Ils étaient tellement différents !

Son père portait les prénoms de Léon, Baptiste, Hubert. C'était un homme bon, profondément bon. Grand travailleur, il était doué d'un courage étonnant. Rien ne l'arrêtait. Son père disait de lui : « Mon grand (parce qu'il était assez grand), mon grand n’est jamais fatigué !»

Sa mère, Flore Dugnolle, originaire du pays d'Ath, était aussi une femme forte. Superintelligente, elle savait tout faire : cuisine variée, pâtisseries, couture. C'est elle qui taillait les costumes de ses fils et qui, plus tard coudra les soutanes de Robert... Quand elle tombera malade, atteinte d'un cancer qui l'emporta en six ou sept mois, elle devint, au grand étonnement de tous, d'une douceur et d'une patience qu'on ne lui avait jamais connues. 
Robert était très attaché à son père, qui l'a fort marqué. Lui, qui a toujours aimé la solitude des grands espaces, adorait, pendant ses vacances aller aux champs pour prendre part aux travaux agricoles : labourer, faire les moissons, battre le blé. Ni la charrue ni la herse ni le fléau n'avaient de secret pour lui. Devenu prêtre, il continuera, en soutane, à aider son père aux labours. Il savait que labour et labeur ont la même origine et que passer du travail sacerdotal au travail de la terre, c'était d'une certaine manière creuser le même sillon. Le métier d'agriculteur comme celui du prêtre demande patience et espérance. L'agriculteur n'est pas maître du climat : il ne fait ni pleuvoir ni briller le soleil. Il doit faire confiance et compter sur autre chose que son propre travail. Sur l'Autre. Sur Celui qui seul fait germer et fait crô̂tre.

Ses rapports avec sa mère étaient plus distants. C'était une femme très énergique, plus dure et plus autoritaire que son père. À l'égard de l'un comme de l'autre, il avait plutôt une attitude de réserve, dans un univers agricole qui, par nature, n'est pas fort loquace et où l'on se vouvoyait en famille, sauf en cas de dispute ! On mangeait ensemble mais on n'entrait pas dans beaucoup de confidences. On allait à tous les offices religieux ensemble, mais on parlait vraiment très, très peu de religion. On était cependant très croyants dans la famille de Robert. Son père était un des seuls hommes du village à aller aux vêpres le dimanche. Il se souvient aussi que, dans sa toute petite enfance, à la mort de son grand-père paternel, on récita le chapelet pendant plusieurs jours. 
Ses parents eurent quatre garçons. Pas de fille. L'aîné, Maurice, mourut de la mort subite des nouveau-nés. Robert, qui le suivit, fut donc l'aîné des vivants : François et Maurice. En effet, on donna au cadet le nom de Maurice, en souvenir de l'aîné décédé prématurément. Robert joua beaucoup avec ses deux jeunes frères. Ainsi qu'avec les gamins et gamines du village. Les jeux dangereux ne leur faisaient pas peur. Un jour, ils se risquèrent à passer entre les bras en mouvement d'un moulin à vent, qui n'était pas loin de leur maison. Une petite Irma fut blessée à la tête. Elle eut plus de peur que de mal, mais l'accident les impressionna fort. Robert se souvient aussi avoir vagabondé avec un de ses frères, à travers champs et bois. Ils ne rentrèrent que fort tard ce jour-là. De part et d'autre de la porte d'entrée de la maison, deux baguettes les attendaient : une pour chacun. Cela leur annonçait très symboliquement la façon dont ils allaient être reçus!

Robert admirait beaucoup son grand-père maternel, François Dugnolle, qui avait un vrai tempérament d'intellectuel. Il raconta à son petit-fils que, tous les dimanches, quand, encore gamin, il allait à pied à la messe à Tournai (vingt kilomètres aller, vingt kilomètres retour !), il s'arrêtait dans une librairie pour s'acheter des livres. Toute sa vie cet homme a beaucoup lu. Peu avant de mourir, en quarante, il lut le Mein Kampf d'Adolf Hitler et la thèse de doctorat en théologie, que Robert Guelluy défendit en septembre. Il avait quatre-vingt-quatre ans. Cet homme était très écouté dans le village. On allait le consulter pour tout. Il faisait même fonction de vétérinaire. De plus, il était ré- 
puté pour détenir divers secrets de fabrication, dont un procédé pour aiguiser les meules de moulin.

\section{Ses débuts à l'école et sa première colère}

Ses parents trouvèrent Robert intelligent. Aussi l'envoyèrent-ils à l'école dès trois ans, alors qu'à l'époque on ne commençait d'habitude que vers cinq ans. Son plus ancien souvenir c'est un tableau représentant végétaux et animaux avec une évocation de Dieu qui trônait toutpuissant au milieu de sa création.

Ses premières années remontent à la guerre de 14-18, la « grande guerre » comme on l'appelait. Des soldats allemands étaient logés chez l'habitant dans des maisons ou parties de maisons réquisitionnées. Partout, il y avait des « chambrées ». Chez les Guelluy, était hébergé un militaire allemand, d'origine polonaise. C'était un doux, qui recevait volontiers le petit Robert. Ses parents étaient très mécontents de voir que leur fils se plaisait en la compagnie de cet homme. Un soldat allemand ça ne se fréquentait pas en ce temps-là !

Il devait avoir trois ou quatre ans, peut-être cinq, quand il piqua la première colère violente dont il se souvienne. Un beau matin, il s'était plu à dessiner un vélo. Mais, à l'endroit du pédalier, il avait omis de dessiner les rayons, voulant éviter de les empêtrer dans les lignes du pédalier. Les adultes auxquels il montra son œuvre lui en firent le reproche. Il se mit alors dans une colère noire, considérant que 
ces adultes n'avaient vraiment rien compris à rien. Cela le marqua très fort et sa colère ne fut pas passagère. À midi, quand il alla dîner chez son parrain (Jean-Baptiste Guelluy, frère aîné de son père), il n'était pas encore refroidi ! Ce fut la première mais pas la dernière de ses grandes colères. Il se souvient aussi d'un différend qui l'avait opposé à son père. La tension monta à un tel point qu'il dut se sauver en courant, mais il fut vite rattrapé à la course.

À six ans, il passe à l'école primaire. Un des souvenirs qu'il garde de cette période c'est que lorsqu'un de ses instituteurs se maria, c'est lui qui fut choisi pour prononcer le discours de circonstance qui avait été composé à cette occasion. Il s'en sortit honorablement, alors même qu'il avoue n'avoir absolument rien compris à ce qu'on lui avait appris à lire !

\section{Ses premières expériences religieuses}

Dès l'âge de sept ans, Robert devient enfant de chœur. Cette fonction impliquait un lever matinal et un départ rapide. Dans ses sabots de bois, dont à quatre-vingt-cinq ans il entend encore le claquement sur le pavé, il s'en allait donc, été comme hiver, servir la messe du matin. Une tournante réglait le service des enfants de chœur. Chacun avait sa semaine.

Il fut assez impressionné par ce qu'on appelait alors des « missions ». Un prédicateur appartenant à un ordre religieux venait pen- 
dant quelques jours dans la paroisse pour donner une sorte de retraite collective. C'étaient des moments forts pour la vie de foi.

Vers dix ans, il se préparait à sa communion solennelle. Lors d'une des instructions, il fut particulièrement marqué par la leçon de catéchisme. Il ne se souvient plus du sujet de cet enseignement, mais cela l'impressionna au point qu'il remonta à la maison dans un recueillement intense et profond. Ce fut pour lui un moment d'expérience religieuse inoubliable, dont l'orientation de toute sa vie dépendra. Comme professeur de séminaire, il eut l'occasion de suivre le cheminement de nombreuses vocations. Il put constater que tous les jeunes adolescents qui optent pour le sacerdoce ont vécu une expérience d'enfant, indicible peut-être mais déterminante. Si rien ne se passe pendant l'enfance, rien ne se passe dans la suite.

\section{Robert entre au collège}

Après ses classes primaires, il entre, comme interne, au Collège Notre-Dame de Kain-la-Tombe, fondé au début du 19e siècle par un prêtre français. Son frère François alla, lui, au Collège de Renaix (en flamand : Ronse) où se côtoyaient flamands et francophones. C'était plus près. Il n'était pas interne : il faisait la route en vélo. Maurice, le plus jeune, fut inscrit à l'école agricole de Leuze. François dut arrêter ses études après sa troisième année d'humanités, malgré les supplications de ses professeurs, qui auraient aimé qu'il poursuive. Mais l'oncle François Dugnolle n'avait pas d'enfant, et l'entreprise qu'il diri- 
geait attendait un successeur. C'est au jeune François qu'il reviendra de prendre la relève de cette petite PME, qui employait trois ouvriers. Quant à Maurice, il reprendra la ferme familiale à la mort de leur maman, qui survint peu après son mariage. Seul Robert fut donc destiné à une carrière intellectuelle.

Ses deux frères furent tous deux des membres actifs de la JOC, la jeunesse ouvrière catholique, mouvement animé par le vicaire du village. Robert étant à l'internat s'y est peu engagé. En fait, c'était une fausse JOC, puisque ce n'étaient pas des ouvriers, mais des patrons et ouvriers agricoles qui en faisaient partie. Plus tard, on créa la JAC, la jeunesse agricole catholique.

\section{Vous n'êtes qu'un paresseux !}

Le collège c'était un autre monde. Trois cent dix élèves ! Une douzaine de professeurs, tous prêtres, auxquels on pouvait se confier, se livrer... Robert lisait beaucoup. Il se souvient particulièrement d'un livre sur les esquimaux, qui a souvent et longuement retenu son attention. Mais ce grand lecteur était plutôt fainéant. Son maître de sixième latine, l'abbé Roland, lui dit : " C'est pas permis, vous devriez être premier de classe ! Vous n'êtes qu'un paresseux ! » Mais le petit Robert ne comprenait pas et trouvait ça très injuste d'être traité de la sorte. 
En deuxième année d'humanités, il avait douze ans et était toujours aussi paresseux. Mais sans doute n'avait-il pas l'air trop méchant. Puisqu'un professeur l'appela et lui demanda : « N'avez-vous jamais songé à devenir prêtre ? » L'effet de la question a été radical : «Ce professeur-là ne me reverra plus jamais ! Ni lui ni quiconque me parlera de la sorte ! » Et il tint parole.

Les années s'écoulant, de paresseux qu'il était, il devint peu à peu plus actif. Les sciences étaient sa matière favorite. Pendant ses six années d'humanités, il eut toujours le premier prix dans cette branche.

\section{Il devient premier de classe}

Mais ce ne sera qu'à quatorze ans, en quatrième latine, sous l'impulsion de l'abbé Voisin, qu'une maturation subite se produisit en lui. Il se mit alors à prendre sérieusement goût à l'étude. Toutes les cours l'intéressaient. Cependant il continua à privilégier le domaine scientifique qui le passionna plus que jamais. L'abbé Roland avait raison : il avait l'étoffe d'un premier de classe. Voilà qu'il l'était et le restera.

C'est son professeur de troisième latine, la classe qu'on appelait « syntaxe ", qui exerça sur lui le plus de fascination. Homme très silencieux, du milieu rural, profondément chrétien. Bien que Robert n'eut que deux fois une conversation personnelle avec lui, ce maître le marqua de façon déterminante. Rigoureux, savant, il donnait le grec 
avec passion. Mais une passion cachée. Il développa chez Robert le désir du travail et le goût de la recherche. Les versions étaient préparées à l'école et les formes les plus difficiles étaient expliquées par le professeur. Mais Robert mettait ses notes de côté, animé de la volonté de tout retrouver par lui-même. C'est à cette époque qu'il rêvait, lorsqu'il pensait à son avenir, de devenir vétérinaire, pour soigner les animaux et rester vivre à la campagne. Oui, ce fut là son rêve le plus tenace.

\section{Un œil au beurre noir !}

Un jour, l'abbé Deviane, son professeur d'histoire et de géographie, l'avait surpris jouant avec son voisin. Robert proteste vivement quand il est interpellé. Et il refuse de sortir quand son professeur veut le mettre à la porte. Il se met à courir sur les bancs poursuivi par l'abbé Deviane qui cherche à l'attraper pour lui donner une raclée. Cela se termina par une gifle magistrale.

Cette histoire a une suite. Le lendemain, Robert se présenta avec un œil au beurre noir au cours de l'abbé Willems, grand polyglotte et professeur de flamand, dont le cours fut particulièrement chahuté. S'adressant à Robert, il lui dit : «Guelluy, il y a encore de la place de l'autre côté !» 


\section{Sa vocation}

Oui, ce sont plutôt pour lui de bons souvenirs. Il ne gardait aucune rancune de ces mauvais traitements. C'étaient les risques du système ! Mais Robert ne trouvait quand même pas idéal ce genre d'éducation. Aussi, lorsque vers ses seize ans se dessina sa vocation, la première de ses motivations était d'essayer de faire mieux que ses maîtres, d'affiner les méthodes éducatives de l'époque, qui le scandalisaient. D'une certaine façon, c'est dans un esprit de contestation qu'il eut l'idée d'entrer au séminaire. Pour faire « autre chose », pour faire mieux.

Ce qui l'attira aussi au séminaire c'était la réflexion philosophique. Oui, au fond, il est entré au séminaire plus pour des buts intellectuels et éducatifs que pour des buts religieux. Il n'avait au cœur ni beaucoup d'amour de Dieu ni beaucoup d'amour des hommes, mais il était passionné par la recherche du sens de la vie. Il lui semblait que c'était en devenant prêtre qu'il se consacrerait le mieux à scruter la signification de l'existence humaine.

Un seul de ses maîtres se distinguait des autres, tous âgés et relativement distants. C'était l'abbé Sironval, titulaire de poésie. Il venait d'arriver au collège et représentait une nouvelle génération, beaucoup plus proche des jeunes. Il jouait au football avec les élèves. En

soutane ! Il exerça vite une grosse influence par son dynamisme, sa 
cordialité et sa familiarité. Sa chaleur humaine le rendait très sympathique. Aussi est-ce à lui qu'au cours du troisième trimestre de sa rhéto, entre Pâques et Pentecôte, Robert ira se confier, lorsqu'il sentit nâ̂tre en lui sa vocation. La réaction de l'abbé Sironval fut directe et toute simple : «Je crois que c'est bien; je célébrerai la messe pour toi demain. »

Ce ne fut pas une vocation tout à fait radicale. Une certaine hésitation subsistait. Ce n'est qu'à la Noël 1930, à la fin de son premier trimestre à l'Institut Supérieur de Philosophie, à Louvain, qu'il écrira dans ses notes spirituelles : «Maintenant je suis certain de ma vocation! \C'est dire que tout ce premier trimestre il resta dans l'expectative.

\section{Robert et les filles}

Cette hésitation était d'ordre intellectuel. Elle n'était pas liée à un problème d'affectivité ou de sexualité. Robert n'avait pas eu de sœur. L'école primaire des filles était tout à fait distincte de celle des garçons. Tout était fait pour que filles et garçons ne se rencontrent pas, sauf au catéchisme. Au collège, non plus il n'y avait pas de filles. La mixité n'existait pas à l'époque. Et à l'université, les étudiantes étaient toutes placées d'office aux premiers bancs : il n'a jamais conversé avec aucune d'elles et pas une ne lui laisse le moindre souvenir. Sauf la nièce du chanoine Harmignie. Elle habitait Charleroi. Elle arrivait 
toujours en retard, le lundi. Son entrée était saluée par des applaudissements sur tous les bancs.

Il en fallait alors du courage pour être fille, à l'université ! Les étudiants de médecine étaient les plus bruyants et les plus « guindailleurs ». Les philosophes avaient des cours communs avec eux. Pendant les inter-cours, les garçons jetaient du maïs aux poules : c'étaient les filles des premiers rangs! Ce n'était pas très relevé, mais c'était le mode des relations entre étudiants et étudiantes, en ce tempslà.

Robert a donc vécu et grandi dans un milieu de garçons. Aucune éducation sexuelle n'était prévue, pas plus pour les adolescents que pour les futurs prêtres et futurs religieux. Cela ne lui a vraiment pas fait problème. Non. Il se souvient qu'un soir, alors qu'encore étudiant il participait au grand congrès catholique de Malines, il vit passer un groupe de quelques jeunes filles, qui n'étaient pas mal du tout. Il s'est dit : elles sont très bien, mais ce n'est pas pour moi ! (rire) Sa ferme résolution de Noël 1930 ne fut jamais remise en question. Pour lui, le chemin fut tout droit, tel qu'il en avait décidé.

\section{Le séminaire et l'université}

Sur les dix-neuf élèves de sa rhétorique, quatre sont entrés au séminaire. Il fut le seul de son diocèse à être envoyé à l'université. C'était la voie réservée aux mieux doués. Il quitta donc Kain-la- 
Tombe pour entrer en octobre 1930 au séminaire Léon XIII à Louvain. Ils étaient une vingtaine d'inscrits à l'Institut Supérieur de Philosophie. Leur conduite spirituelle était confiée à un saint homme : le chanoine Brohée. C'est avec lui qu'il apprit peu à peu la méditation, le recueillement, la vie intérieure. C'était un univers très neuf pour lui. Mais il se sentit tout de suite « enchanté », « comme un poisson dans l'eau ».

L'environnement humain était très différent de celui du collège. À Kain-la-Tombe, tout le monde appartenait au même milieu, tout le monde se connaissait, venant de la même localité ou des villages avoisinants. L'atmosphère était très cordiale, très chaleureuse. On s'aimait beaucoup entre collégiens. On montait beaucoup blagues ensemble. À Louvain, l'atmosphère était plus froide, les rapports plus guindés. Ses condisciples venaient d'un peu tous les coins du pays. L'enseignement était plus théorique et certains cours étaient bilingues. Il y avait trois mille étudiants à l'époque, flamands et wallons confondus, et seulement quelques dizaines de filles. Les étudiants ne rentraient en famille qu'environ une fois par mois. Les autres week-ends ils les passaient sur place.

\section{Sa rage au travail et son goût pour les sciences}

Grand solitaire, se contentant de ses livres, Robert fut à l'université un étudiant travailleur. Comme cours à option, il choisissait toujours les plus difficiles : les mathématiques, les sciences et la phy- 
sique spéciale, donnée par un maître marquant de l'époque : le professeur Mund. Lui, qui autrefois avait pensé devenir vétérinaire, était donc toujours passionné par le domaine scientifique. Il fera d'ailleurs un mémoire de licence en critique des sciences. La variété de ses intérêts intellectuels était déjà un signe avant-coureur de son grand souci d'interdisciplinarité... Ce ne sera que plus tard, qu'il commencera à s'intéresser à la Bible, puis à la dévorer. Car, à l'époque, si étrange que cela puisse paraître, il y avait moyen d'être docteur en théologie, sans avoir beaucoup fréquenté l'Écriture ! Ce n'est qu'après la guerre que se sont développés les mouvements bibliques.

Il se laissa prendre par une telle rage au travail que, pour mobiliser au maximum ses potentialités, il s'astreignit à des exercices respiratoires profonds et répétés. Si bien qu'il fut atteint d'une pleurite due, en partie, à ses excès d'étude. Or, à cette époque, la tuberculose était fort répandue et cause de nombreux décès chez les jeunes. Cette inflammation de la plèvre dont il souffrit lui valut d'être réformé en 1932, à la fin de son service militaire. Le médecin qui l'avait examiné voulait, par cette décision, lui éviter une quantité de rappels. Cela signifiait clairement que Robert était « un oiseau pour le chat »! Il reconnaît lui-même que, "par bêtise ", il se tuait au travail : fatigue, tension trop élevée, insomnies étaient son lot quotidien. Mais il était heureux. Heureux en famille. Heureux au collège. Heureux à l'université et au séminaire. Il eut la chance de faire toujours ce qui l'intéressait, ce qui lui plaisait. Et en réussissant ! 


\section{De bonnes blagues!}

Friand d'anecdotes et de bonnes blagues, il se plaisait à rappeler celle-ci remontant au jour de sa démobilisation. Le sergent responsable du stockage des vêtements militaires était bien en peine. Il lui manquait une paire de bottines dans son inventaire. Aussi, pour se tirer facilement d'embarras, ne trouva-t-il pas mieux d'inscrire dans le carnet militaire du soldat Guelluy : «Rentré dans son foyer avec une paire de bottines »! Robert n'était évidemment pour rien dans cette disparition. Il rentra donc chez lui et, comme il était tout de même réellement malade, il prit un peu de repos.

C'est vers la même époque qu'il assista à la première conférence du professeur Georges Lemaître sur son hypothèse du choc de l'atome initial. Cette théorie faisait fort impression. Mais ce grand savant aimait la jeunesse et se plaisait à blaguer avec ses étudiants. Le chahut à son cours ne le dérangeait vraiment pas et parfois il semblait y contribuer lui-même ! Ses cours étaient très sympathiques car l'homme était très sympathique. C'est dans ce climat-là de bonne humeur que quelqu'un, sans méchanceté aucune, avait ajouté sur la porte de son bureau le préfixe « sur » devant le titre « chargé de cours », ce qui donnait « surchargé de cours à l'université »! Il est resté très populaire pendant toutes les années de son enseignement. 


\section{L'avant-guerre et l'aventure rexiste}

Quand, en 1934, Robert Guelluy obtient sa licence en philosophie thomiste, il retourne dans son diocèse, au Grand Séminaire de Tournai, pour sa formation fondamentale en théologie. Il sera ordonné le 20 février 1937. Mais depuis octobre 1936, il était à nouveau à Louvain pour la suite de ses études à la « schola maior » de théologie et pour préparer sa thèse doctorale, qu'il défendra en septembre 1940.

À l'université, il eut comme compagnon le fils du roi d'Espagne et un certain Léon Degrelle... À ses débuts, on ne pouvait prévoir l'évolution du mouvement rexiste. Léon Degrelle commença comme président de l'A.C.J.B., l'Action catholique de la jeunesse belge. Il était très lié à l'Église et n'a pas hésité d'affirmer : "Pour nous, un désir des évêques c'est un ordre. » Il fut fortement encouragé et appuyé par Monseigneur Picard, aumônier de cette toute neuve Action catholique.

Léon Degrelle était un mégalomane qui vivait d'un rêve : le retour aux États bourguignons de la fin du moyen-âge. C'est cela qui représentait, à ses yeux, le destin futur de l'Europe. Cependant ce qui a prévalu pour lui, ce fut moins cet enthousiasme européen que l'aspiration à une société, à un gouvernement, à une classe dirigeante purifiés. Les autorités en place se voyaient accusées d'être minées par la corruption. Le Pays réel, journal du mouvement rexiste, dénonçait 
tout ce qui était malsain dans la politique du pouvoir. Beaucoup de jeunes que fréquentait Robert Guelluy étaient séduits par ce thème. Grâce à ses dons incontestables d'orateur et de tribun, Degrelle parvint à susciter un enthousiasme extraordinaire chez cette génération montante, idéaliste et un peu utopiste, qui rêvait d'une société complètement assainie.

Le Professeur Carnoy, linguiste chargé de l'enseignement du grec à la Faculté de philosophie et lettres, fut particulièrement visé par les sarcasmes et les accusations de Léon Degrelle. Il faut savoir qu'Albert Carnoy avait été éphémèrement ministre dans un des gouvernements d'avant-guerre. La rumeur publique raconte que lorsqu'on lui téléphona de nuit pour lui proposer ce poste, c'est sa femme qui décrocha et qui, sans le réveiller, répondit aussitôt : " Nous acceptons ! » Un jour, une des filles Carnoy, qui assistait à un meeting rexiste, fut tellement excédée par les critiques dont son père était la cible qu'elle monta sur le podium et gifla Léon Degrelle en public. Évidemment, cela fit sensation!

Lorsque le rexisme vira au nazisme, beaucoup quittèrent ses rangs et il ne recruta plus dès lors que dans les milieux d'extrêmedroite, où il y avait moins d'idéal que de rage au cœur. Consciente que ce mouvement devenait menaçant pour l'avenir du pays, l'Église pesa de tout son poids pour endiguer la vague rexiste et précipiter sa chute. La guerre éclate 
Lorsque la guerre éclate, Robert Guelluy mettait la dernière main à sa thèse de doctorat. Il passa la nuit du 9 au 10 mai 1940, à corriger, avec son ami Arthur Gille (qui deviendra professeur de pédagogie), le texte dactylographié en cinq exemplaires sur du papier pelure avec des carbones, comme cela se faisait à l'époque. C'est le cinquième exemplaire, le moins clair, que son grand-père lira avant de mourir. Les machines à écrire ne mettaient pas d'accent sur les lettres majuscules, comme peuvent le faire les traitements de texte d'aujourd'hui. Mais les exigences des examinateurs étaient grandes. Aussi toute cette nuit se passa à mettre des accents sur tous les E majuscules où il en fallait! Et cela, à la lumière d'une bougie, car au Collège du Saint Esprit l'électricité était coupée à 22 heures et la clef de la maison mise sous l'oreiller du gérant ! Le travail ne se termina qu'à l'aube et il ne lui resta qu'une demi-heure de sommeil, avant d'aller célébrer la messe dans le couvent voisin des sœurs dominicaines, qui géraient un petit home pour personnes âgées. Durant son court sommeil, il rêva que c'était la guerre et que le roi Léopold III avait fait, comme son père, un discours devant le parlement. Mais Léopold III était déjà en froid avec le régime parlementaire et ce discours n'eut pas lieu. De Louvain, on entendait déjà les bombes qui tombaient sur le terrain d'aviation militaire pas très éloigné. À la fin de la messe, lorsqu'il sortit de chez les sœurs dominicaines, il vit son patron de thèse, le chanoine De Meyer qui descendait en courant la rue de Namur, où il avait célébré la messe à la pédagogie féminine Sedes Sapientiae. Il courait pour le rattraper. Et il lui dit: «Vous savez, c'est la guerre !» 
Comme il avait été réformé en 1932, il se rendit immédiatement à son évêché. Monseigneur Delmotte décida qu'il ne devait pas s'engager mais essayer de remplacer dans leurs tâches les prêtres mobilisés. Le diocèse en avait grand besoin. C'est ainsi qu'il fut nommé provisoirement vicaire à la paroisse Saint-Brice de Tournai, dont le vicaire, l'abbé Chaudière, était mobilisé. Il occupa donc la maison de l'abbé Chaudière, près de l'église, mais avait reçu la consigne d'aller loger au séminaire. D'ailleurs, dans cette maison, seul le bureau lui était accessible. Toutes les autres portes étaient fermées à clé.

\section{Tournai sous les bombes}

Dans l'après-midi du 16 mai, il était dans son bureau lorsqu'une femme sonna pour demander conseil. Devait-elle tenter de rejoindre à Mons son mari, militaire de carrière mobilisé ? Il se souvient lui avoir répondu que Tournai était une zone de sécurité et que ce serait risqué pour elle d'aller jusqu'à Mons. On voyait défiler dans les rues les réfugiés venant de tous les coins du pays : ceux de la frontière allemande, ceux de Verviers, puis ceux de Liège, de Bruxelles... On aurait dit que peu à peu c'était le pays entier qui était déserté. Lorsque les bombes se sont mises à tomber en sifflant, la première réaction de Robert fut de courir au jardin, se disant : « Si la maison est écrasée, au moins je ne serais pas en dessous ! » Puis, se ravisant, il crut plus sage de rejoindre l'abri public aménagé près de l'église Sainte-Brice, à deux cents mètres de là. Quand il ouvrit la porte pour sortir, il se heurta à trois hommes réfugiés sous le porche, qui s'engouffrèrent dans le 
hall et coururent directement vers le fond du couloir pour se précipiter dans la cave. Mais la cave était aussi fermée et il n'en avait pas la clé. Il engagea donc ces trois hommes à faire comme lui et à se rendre à l'abri public. À ce moment-là une bombe tomba à un mètre cinquante d'eux, sur le trottoir, en face de la fenêtre de la maison. Il fut blessé à la main. C'était inquiétant! On entendait les sifflements des bombes des escadrilles allemandes qui suivaient l'Escaut, traversant la ville de Tournai dans le sens du parcours du fleuve.

Ces bombardements sont décrits dans un volume paru quelques années après la guerre, intitulé Tournai sous les bombes. En le lisant, Robert apprit que la bombe tombée sous ses yeux faisait partie d'un lot de petites bombes de cinquante kilos, conçues non pas pour tuer la population mais pour abattre les immeubles. Elles ne produisaient pas d'éclats en hauteur mais elles éclataient dans le fond de leur trou. Il se souvient des nuages de cailloux qui s'élevèrent devant lui et des énormes bordures de trottoir littéralement soufflées. L'une lui passa au-dessus de la tête et atterrit sur le toit de sa maison. Une autre s'écrasa dans le grenier du doyen, dont l'habitation était voisine.

Arrivé en courant à l'entrée de l'abri, il tenta d'écarter du pied un chien qui aboyait avec angoisse face à tout ce monde encaqué. Cela suscita protestation de la maîtresse du chien qui n'avait à cœur que la protection de son animal!

Quand le bombardement se calma, il sortit de la mêlée et vit des tas de décombres. Il donna des absolutions collectives, comme cela 
était prévu en cas d'urgence, lorsqu'il voyait dépasser des membres de personnes décédées sous les gravats. Deux chevaux de la brasserie $L e$ Lion aussi avaient péri. Dans le courant de la journée, lorsque les bombardements reprirent, l'église fut atteinte et commença à brûler. Il aida à évacuer les objets en péril. Les bombardements et l'incendie durèrent toute la nuit.

\section{L'exode}

Le lendemain, l'occupation allemande étant prévisible, l'évêché décida que les jeunes devaient partir pour échapper autant que possible à la captivité. En ce temps-là, l'utilisation du vélo était interdite aux prêtres et aux séminaristes par l'évêché de Tournai. Tout moyen de locomotion était d'ailleurs proscrit, parce que l'évêque, Monseigneur Rasneur, voulait que les prêtres aillent à pied pour multiplier les occasions de contact avec les paroissiens. Mais, « à la guerre comme à la guerre » (c'était vraiment le cas de le dire), Robert alla dans sa famille, récupérer le vieux vélo de sa mère, donc un vélo de femme, pour se mettre en route tout de suite. Il prit soin d'amener avec lui sa thèse de doctorat qu'il n'avait pas encore défendue et la ficela sur son porte-bagages. Il enfourcha son vélo et partit en direction de la frontière française. Des colonnes de Tournaisiens évacuèrent ainsi leur ville, privée d'électricité, pour fuir les bombardements et dans la crainte de l'arrivée des Allemands. 
Au sortir de Tournai, un jeune homme d'Enghien ainsi qu'un collégien, élève chez les joséphites à Anvers, lui demandèrent s'ils pouvaient se joindre à lui pour la route. Il perdit rapidement de vue le collégien, tandis que le jeune homme l'accompagna jusqu'au village de Berk Plage où ils arrivèrent le lendemain. Il rencontra là le vicaire de son village natal, Saint-Sauveur, qui célébrait la messe au moment où il entrait dans l'église, aussi dans le but de la célébrer. Il y avait déjà, à ce moment-là, des soldats allemands sur la grand-route de Berk Plage. Il en interrogea un qui lui conseilla de faire la route qu'euxmêmes venaient de parcourir, mais en sens contraire : c'est-à-dire dans la direction d'Arras. Sur cette route, il fut rattrapé par un jeune Père Blanc, accompagné de sa sœur, toute de noir vêtue, en deuil de leur père mort récemment. Les Allemands réquisitionnaient tous les hommes célibataires qu'ils rencontraient pour les utiliser à différentes tâches. Par exemple au nettoyage des mairies et autres bâtiments où ils comptaient s'installer. Croyant qu'ils avaient à faire à un jeune ménage, ils poussèrent le jeune Père Blanc (qui avait été formé à l'obéissance !) avec sa sœur, dans la prairie toute proche, en disant : «Allez dans le jardin ! ». Robert profita de la confusion pour s'éclipser d'un vigoureux coup de pédale et continuer son chemin en vélo.

Arrivé dans un petit village près d'Arras, dont il a oublié le nom, il fut arrêté par des soldats allemands. Ils fouillaient tous les réfugiés parce que, à Arras, avait eu lieu une tentative de contre-offensive anglaise visant à freiner l'avance du flux envahisseur. C'est dans ce petit village qu'il séjourna, hébergé par le jeune curé de l'endroit. Celui-ci était en charge de deux paroisses. Il en confia une à Robert. 
Il entendit beaucoup de confessions durant ce court séjour. Il en a gardé un souvenir tout particulier parce que leurs thèmes n'étaient pas du tout habituels. Sans trahir aucun secret, il peut dire qu'il était confronté à bien des questionnements d'hommes qui remettaient en cause certains comportements qui avaient orienté leur vie. Tel ce fonctionnaire qui avait payé des employés de l'administration pour obtenir le poste qu'il ambitionnait et occupait depuis bien des années. Ou tel cet administrateur-délégué d'une firme industrielle qui touchait de gros dividendes pas très réguliers. Ce ne sont pas les cours du séminaire qui préparent à affronter des problèmes aussi concrets et aussi vitaux!

\section{Retour après l'exil}

Lorsque, le 28 mai, il apprit par la radio la capitulation de l'armée belge, il décida de mettre fin à son exil français et de rentrer aussitôt. Il se mit en route vers Mons et, de là, regagna Tournai.

Après son bref exode, il reprit ses fonctions de vicaire de Sainte-Brice, dans l'hôpital des Sœurs de la Charité de Gand, où il restaura le culte interrompu depuis le 16 mai. Il remplaça aussi, durant sa courte absence, le doyen de Tournai, qui était parti pas fort loin pour mettre ses parents à l'abri. 
Un jour, entre le séminaire où il logeait et la chapelle où il allait célébrer la messe, il fut arrêté par une auto de fonctionnaires du ministère des affaires étrangères d'Italie. Ils lui apprirent que leur pays venait d'entrer en guerre. La reine d'Italie, qui était la princesse MarieJosée de Belgique, voulait avoir des nouvelles de sa tante, religieuse au couvent qu'on appelait Le Coquelet à Namur. Robert s'informa. On lui conseilla d'aller voir à 1'Hôpital des Sœurs Bretonnes où, selon la rumeur publique, s'abritaient beaucoup de réfugiés. Effectivement, cette religieuse, sœur de sang du roi Albert, y était passée la veille, mais avait pu partir vers la France avec le flot des réfugiés de Tournai. Il apprit, après la guerre, qu'elle avait réussi à traverser la frontière avec sa communauté et qu'elle avait abouti dans le Midi.

Quelques séminaristes de Tournai réformés, étaient parvenus à échapper aux Allemands qui voulaient regrouper tous les jeunes dans un centre en France. Accompagnés par l'abbé Samain, ancien compagnon de classe de Robert et qui, par la suite, deviendra évêque auxiliaire, ils réussirent vaille que vaille à réorganiser les cours. Robert leur dispensa pendant deux semaines un cours sur la foi, en remplacement de l'abbé Thomas, le futur Monseigneur Thomas, Vicaire général. Robert ne savait pas alors que sa destinée l'appellerait à une longue carrière dans ce séminaire, qui lui a toujours été si cher. 


\section{Une thèse de doctorat comme oreiller !}

Il ne perdait pas, pour autant, de vue sa thèse doctorale qu'il aurait dû déposer à sa Faculté à Louvain dans l'après-midi du vendredi 10 mai. Elle l'avait accompagné, ficelée sur le porte-bagages de son vélo, durant son exode. Et plus d'une fois elle lui avait servi d'oreiller dans les logements de fortune de son périple de réfugié ! Il manquait encore une page à cette thèse : la dernière page de la conclusion, qui était toujours à la dactylographie. Il remonta donc à Louvain pour la récupérer.

Il déposa sa thèse dès que la Faculté fut réouverte, après les bombardements de Louvain, et la défendit en septembre. Mais il lui restait quelques heures de cours à suivre pour achever son programme doctoral. Ils eurent lieu en septembre-octobre.

D'une façon symptomatique, l'abbé Guelluy note dans sa thèse que les théologiens louvanistes de la première moitié du XVIe siècle « s'attachent à défendre l'authentique notion de foi plutôt qu'à y faire voir une rencontre personnelle du croyant avec Dieu », alors que ceux de la seconde moitié du XVIe siècle voudraient que « la théologie de leur temps soit plus fidèle à l'Écriture et parle davantage au cœur ». Pointe déjà ici cette préoccupation essentielle de Robert d'une relation vivante du fidèle avec son Dieu. 


\section{Vers la maîtrise}

Licencié en philosophie thomiste en 1934. Prêtre en 1937. Docteur en théologie en 1940. Robert Guelluy ne va pas s'arrêter en si bonne voie. Le voilà qui s'attaque à une maîtrise en théologie, grade équivalent de l'agrégation de l'enseignement supérieur. La bibliothèque venait de brûler. Il lui faut donc trouver un sujet tout neuf, que personne ou à peu près n'avait encore abordé, afin d'éviter l'écueil de la bibliographie. Les circonstances le poussent ainsi à étudier un franciscain du XIVe siècle, assez ardu : Guillaume d'Ockham. Il s'en alla à Paris consulter le grand médiéviste, Étienne Gilson, qui fumait sa pipe toutes les après-midis, à la librairie Vrin. Quand il lui a exprimé son embarras, lui disant qu'il ne comprenait rien à son auteur, Étienne Gilson lui dit : «Tranquillisez-vous, moi je ne comprends rien à Duns Scot et je dois faire cours sur Duns Scot dans deux mois. " Ainsi armé, il se mit à relire Guillaume d'Ockham. Et c'est alors que s'est produit un grand choc, un bouleversement qui a marqué toute sa vie. Il venait de découvrir la clé de cette pensée. C'était absolument hallucinant. Dieu est libre à ce point qu'avec lui plus rien n'est sûr. À tout instant tout peut arriver. Alors que l'homme aime quelqu'un dans la mesure où il est aimable, Dieu aime sans aucun préalable. Je n'ai aucun titre à faire prévaloir. Je suis aimé tel que je suis. Cette libre initiative de Dieu c'est un des noms de l'amour. Dieu est libre parce qu'il n'est rien d'autre qu'amour. 
C'est ainsi que Robert Guelluy comprit que le premier mot et le dernier mot de toute vie c'est un don gratuit. Et que, à sa dernière heure, sa seule sécurité, sa seule paix, sa seule force serait d'être livré à la liberté divine sans arme, sans argument, sans rien à faire valoir. Être libéré de toutes ses vertus et de toutes ses fautes, quel soulagement ! quelle dédramatisation!

\section{Souvenirs de la résistance}

Pendant l'occupation allemande, son frère François, qui était devenu secrétaire communal de Saint-Sauveur, dut plus d'une fois affronter des situations difficiles. Une nuit, alors que tous deux écoutaient, comme chaque jour, la radio branchée sur le poste de Londres, la gestapo survint exigeant l'adresse d'un certain Roger Cantraine, chef de résistance. C'était un homme de lettres, professeur de français. Il a écrit plusieurs livres de récits se passant dans le milieu rural du Tournaisis. À l'époque, il avait vingt ans. Usant de ruse, François répondit : "Moi, je suis secrétaire communal, je ne connais que les papiers. Allez trouver le garde champêtre, qui, lui, connaît bien le village. » Une fois la gestapo partie, Robert et François se concertèrent et mirent au point une stratégie pour permettre à Cantraine de prendre le large. François téléphona au garde champêtre, lui donnant comme consigne d'égarer la gestapo en lui indiquant des chemins détournés. Il prévint aussitôt Cantraine du danger qui le guettait. Celui-ci eut ainsi le temps de prendre la fuite et d'avoir la vie sauve. 
François s'illustra aussi en une autre occasion. Il avait appris qu'avaient été dressées des listes de jeunes gens et de jeunes filles qui étaient sur le point d'être réquisitionnés pour le travail forcé en Allemangne. De nuit, il s'introduisit à la Maison communale, en brisant la vitre d'une fenêtre. Il s'empara de ces listes et les détruisit. Grâce à lui, ces jeunes échappèrent donc à la réquisition, puisque les listes avaient disparu. C'était évidemment une opération à haut risque !

Maurice, le plus jeune des frères Guelluy, celui qui a repris la ferme familiale, avait dix-neuf ans quand il a été mobilisé en 1940. On emmenait en France, en colonnes, les jeunes pour y être formés militairement. La colonne de Maurice fut prise pour cible dans le nord de la France par un avion anglais qui la bombarda, croyant qu'il avait affaire à des militaires allemands. Quand il fut touché, Maurice tomba dans le coma. Tous ceux qui étaient saufs s'étant enfuis, lorsqu'il revint à lui, il se trouva seul, entre la vie et la mort, au milieu d'un désert de cadavres. Un éclat d'obus lui avait traversé l'os iliaque et s'était logé dans le ventre, tout près des nerfs de la colonne vertébrale. C'est dans un hôpital de campagne, à Lophem, où il avait été transféré, que Robert, qui était à sa recherche, le retrouva. Son état était très critique et il dut rester dix mois hospitalisé. Chaque jour, on extrayait de sa plaie près d'un litre de pus. Après son hospitalisation, il fut réformé et put rentrer à la ferme. Les juments, rebelles pendant toute son absence, reconnurent immédiatement leur maître et se laissèrent mettre le harnais. 


\section{La fin des hostilités}

De 1941 à 1945, la thèse de maîtrise de Robert Guelluy ne progresse pas fort. Faute de documents d'abord, parce que pendant la guerre bien des sources étaient difficilement accessibles. Et faute de temps aussi, car sa charge d'enseignement au Séminaire épiscopale de Tournai l'accaparait beaucoup. Dès la fin des hostilités, il put se consacrer davantage à sa recherche et en deux ans, sous la direction du chanoine F. Van Steenberghen, il rattrapa son retard. En 1947, il publiait sa thèse Philosophie et théologie chez Guillaume d'Ockham. Pendant qu'il achevait cette maîtrise, il suivit quelques cours complémentaires pour obtenir aussi un doctorat en philosophie. C'est une partie de sa thèse de maîtrise qu'il présenta comme thèse de doctorat : La Théorie de la science chez Guillaume d'Ockham. En 1947, il ajoutait ainsi à sa panoplie de diplômes celui de maître agrégé en théologie - qui faisait de lui un « oiseau rare » - et celui de docteur en philosophie. La même année il devenait chanoine honoraire de la Cathédrale de Tournai.

\section{Le plus beau métier du monde}

Comme professeur au séminaire, c'est cent vingt futurs prêtres qu'il a charge de former. Allergique au dogmatisme (parfois même un peu trop !), il renouvelle l'enseignement du Dogme dans un sens plus spirituel que doctrinal. Et ce même souci de rapprocher théologie et 
formation spirituelle on le retrouve dans les cours qu'il dispensa pendant vingt ans à Bruxelles à l'École des sciences philosophiques et religieuses des Facultés universitaires Saint-Louis, entre 1949 et 1969, ainsi qu'à l'Institut international Lumen Vitae de 1959 à 1969. Sa théologie ne fut jamais un savoir sévère et triste, celle des abstractions closes. Pour lui, être professeur de théologie c'est avant tout parler de ses raisons de vivre. Y a-t-il plus beau métier au monde?

\section{Professeur}

à l'Université catholique de Louvain.

À cinquante ans, en 1963, il quitte son cher séminaire de Tournai, où on avait eu le temps de lui préparer un successeur. En effet, il est appelé à la Faculté de théologie de l'Université catholique de Louvain (U.C.L.) pour assurer des cours, dans le cadre d'une licence en sciences catéchétiques qui vient d'être créée à l'Institut supérieur des sciences religieuses. Il inaugure une chaire traitant des rapports entre la foi et les mentalités contemporaines. Il est chargé aussi, entre autres, de questions spéciales sur la doctrine de la vie consacrée et sur la catéchèse de la spiritualité. Lorsqu'en 1969, la Faculté restructure ses programmes, répondant au vœu du chanoine Guelluy, elle crée et lui confie des cours de pastorale, avec ouverture pluridisciplinaire, où sont abordés les thèmes de la rencontre avec l'athéisme, du dialogue avec les milieux scientifiques, et des problèmes spirituels engendrés par la crise économique. À l'occasion de son accession à l'éméritat, en 1984, c'est au chômage des jeunes qu'il demande de consacrer un 
colloque. Le Chômage des jeunes, défi aux chrétiens est le titre de l'ouvrage collectif, couronnant l'engagement social de Robert Guelluy. Le choix de ce sujet est significatif d'une préoccupation constante de cet homme né dans un milieu de travailleurs agricoles et qui, en 1953, publiait déjà, à la demande des mouvements belges d'action catholique rurale Le Travail dans la vie du chrétien. Il y écrivait ces mots qui résument bien toute sa pensée : « le ciel n'est pas étranger à la terre ».

\section{Des figures emblématiques}

Le cardinal Cardijn fut pour lui une figure de proue de la défense de la classe ouvrière. Dès le séminaire, il le fréquenta et eut l'occasion de collaborer avec lui au cours de quelques réunions jocistes. Celles-ci, dans leurs débuts, rassemblaient quelques jeunes ouvriers, autour d'un réverbère. Les dirigeants leur faisaient raconter leur vie, puis réfléchir sur le sens à lui donner. La formule Voir, juger, agir inventée par Cardijn était le secret de la vraie réflexion, de la vraie intelligence. Il ne demandait pas aux chefs jocistes de tenir un carnet d'idées, un recueil de pensées fortes, mais de rassembler des faits vécus par les jeunes ouvriers : à quelles situations étaient-ils confrontés ? de quoi souffraient-ils ? de quelles atteintes à leur dignité étaient-ils victimes ? C'était une sorte d'enquête permanente sous forme de révision de vie, à partir de laquelle s'élaborait un mode de réflexion et de formation. 
Cardijn avait une extraordinaire puissance de tribun. Quand, dans les meetings, il défendait les droits de la classe ouvrière, il avait l'art de mobiliser les foules et s'adressait à elles avec une vigueur et une fougue tout à fait étonnantes. Il était alors entièrement au service de la cause qu'il soutenait. Mais, par nature, cet homme était un doux. Robert Guelluy se souvient l'avoir accompagné à une session de la Jeunesse Ouvrière Catholique., au cours de laquelle Cardijn s'était particulièrement déchaîné. Au retour de celle-ci, dans sa voiture, il était aussitôt redevenu la paix même. Il n'en voulait à personne et jamais ne manifestait la moindre agressivité contre qui que ce soit. Un jour, le Cardinal Van Roey le fit appeler parce que, dans le petit journal de formation La Vie ouvrière, était paru un article vraiment trop virulent. «Écoutez, lui répondit-il, c'est une école ; ils apprennent à écrire. Il ne faut pas leur en vouloir si parfois ils font des faux pas. » Ce qu'il n'avait pas dit c'est que cet article-là justement c'était lui qui l'avait rédigé !

Robert Guelluy aimait évoquer d'autres figures emblématiques qui l'avait marqué. Le Père Léon Van Hoorde, fondateur de la communauté de La Poudrière à Bruxelles, où des hommes et des femmes de toute condition sociale, de toute origine ethnique, de toute culture, de toute opinion philosophique, de tout âge vivent en commun, unis dans le travail, et partagent leurs revenus. Plus célèbre, Don Helder Camara qu'il rencontra plusieurs fois, au Brésil et en Belgique. Notamment quand il fut promu docteur honoris causa à l'U.C.L. C'était encore à Louvain. Il appréciait son engagement politique, son courage 
et sa lucidité, mais pense qu'il espérait le cardinalat et fut déçu de ne pas y avoir accédé.

Ni très royaliste, ni très papiste. Il se demande pourquoi on a voulu faire un saint du roi Albert, ce héros chevalier ! Lors de la crise royale, il ne fut pas du tout léopoldiste. Il prétendait même avoir été le seul prêtre du diocèse de Tournai à voter NON, lors de la consultation populaire. Quant à l'aura créée autour du roi Baudouin, ce n'est pour lui qu'un «montage mythologique ». Il lui est difficile de croire que le pontificat de JeanPaul II soit sous l'inspiration de l'Esprit. Il n'est enthousiaste ni pour le mouvement charismatique ni pour Taizé. Seuls Jean XXIII et Monseigneur Gaillot ont vraiment ses faveurs. Il aimait répéter : «Ah ! De Gaillot, je ne pense que du bien. » Il apprécie son désir d'une Église servante et pauvre ; sa volonté d'une Église plurielle ; son souci d'une plus grande autonomie pour les Évêques.

\section{L'avenir de l'Église et du monde}

Comment voyait-il l'avenir de l'Église et du monde ? Avec Jacques Meert, un des premiers disciples de Cardijn, il pensait que la crise religieuse actuelle tenait à trois choses : excès de centralisation, excès de juridisme et excès d'idéologie (c'est-à-dire de théorie abstraite). Sans doute, ce n'est pas sans déchirement et une véritable tristesse qu'il regardait ces séminaires vides et ces monastères désertés, où autrefois il avait donné tant de retraites. Mais la vie n'est-elle pas peut-être plus authentique dans l'ébranlement actuel des institutions ? 
Aussi est-ce plutôt avec optimisme qu'il jugeait l'évolution de l'homme d'aujourd'hui, qui a tant besoin d'une parole d'espérance. Devant ce que certains considèrent comme un désaxament sexuel de la jeunesse, il se demande s'il y a plus d'excès dans cette liberté qu'il y en avait dans les refoulements, les tabous, les inhibitions, les hypocrisies d'autrefois. Le chrétien de demain ne sera plus une plante de serre surprotégée. Son espoir et sa joie c'était de penser que partout allaient surgir des bourgeons, des rameaux nouveaux et que l'ancienne unité monolithique, que veut sauvegarder la curie romaine, va faire place à une efflorescence de vie déconcertante, déroutante, dont on ne peut déjà prévoir l'ampleur. Chacun devra prendre sa vie en main, assumer ses responsabilités. Vivre c'est aimer. Aimer c'est risquer. Si l'on peut être satisfait d'être tranquille bien au chaud, il y a aussi une joie d'être en plein vent, en pleine tempête. S'acquitter de ses obligations avec exactitude et rigueur, dans une Église bien organisée, c'est de l'histoire ancienne. Aujourd'hui, il faut être heureux de tout ce qui change, de tout ce qui se diversifie, de tout ce qui vit. Ce sont des plantes nouvelles, vigoureuses, qui vont commencer à croître. C'est à une Église d'un autre style que nous sommes appelés. Et ce ne sera pas triste ! L'Évangile d'ailleurs ne nous propose pas comme programme d'être des hommes de devoir, mais il nous dit : « Tu aimeras Dieu et tu aimeras ton prochain comme toi-même, de tout ton cœur. » $\mathrm{Ce}$ risque de l'amour est tout autre chose que crispation sur soi. $\mathrm{Au}$ contraire, il détend, il apaise. Être chrétien, c'est à la fois simple et difficile : être content de Dieu, être content des autres, être content de soi. 


\section{Le secret de la paix}

Au mot amour, qu'il trouvait tellement galvaudé, Robert Guelluy préférait celui d'affection ou de tendresse. Il avait compris que la pire des choses qui puisse lui arriver c'était de se prendre au sérieux (et même dans son âge avancé cela lui arrivait !). Ce sont les autres qu'il faut prendre au sérieux en essayant de les rejoindre là où ils sont. Il ne s'agit pas de faire du sentiment, mais de se mettre tout le temps en disposition de comprendre, en disposition d'accueillir. Apprendre à se désoccuper de soi-même, afin d'être constamment, toutes antennes ouvertes, disponible à l'écoute d'autrui. Être quelqu'un pour quelqu'un. Privilégier les « relations courtes » comme Ricœur les appelle.

Lui qui, par tempérament, était plutôt de nature inquiète, voire colérique, a découvert que c'était là le grand secret de la paix. Quand, vers la fin de sa vie, d'anciens étudiants le rencontraient et lui disaient : « Mais comme vous avez changé !», il répondait : «Ben, oui, que voulez-vous, mes cheveux n'ont pas toujours été gris. J'ai eu le teint plus frais autrefois ! ». Alors eux de rétorquer : « Non ! Ce n'est pas cela. Mais vous êtes devenu bon. » 


\section{Scruter le réel}

Les prestigieux titres académiques dont il était bardé auraient dû l'orienter vers des études pointues. Mais il n'a pas été fort tenté par la carrière de chercheur académique. Il avait entrepris une édition critique d'un auteur de la fin du Moyen âge, Hervé de Nedelec. Il ne l'a pas poursuivie et a préféré confier à l'université toutes ses photos de manuscrits. Aux abstractions il a toujours préféré les faits, le contact avec le vécu, à travers lequel il aimait lire les signes du temps. C'est la destinée qui fit de lui un professeur d'université. Il décrocha ses diplômes juste au moment où on avait besoin de lui. Voilà tout. Il est persuadé que dans nos existences la part de rationalité est vraiment très limitée. Tandis que la part de hasard est énorme. Et c'est dans cette part de hasard que Dieu agit. Il faut donc se garder de toute organisation excessive. Car, à la limite, quand tout est parfaitement réglé, il n’y a plus de place pour la foi.

Il se considérait comme un faux intellectuel, car ce ne sont jamais les livres qui l'ont vraiment intéressé, mais les gens. Il en a pourtant écrit des livres ! Plus d'une douzaine. Surtout pour le large public. Des livres qui, sur la fin, lui ressemblaient de plus en plus. Tous épuisés comme lui (excepté le dernier), aimait-il ironiser. Mais encore plus que par ses écrits et par ses cours, c'est par ses homélies et ses retraites qu'il a rayonné. Et il en a donné des retraites et des sessions dans 
tout l'univers catholique, à l'exception de l'extrême Orient. Europe, Afrique, Amérique du Nord, Amérique du Sud. Il a même donné une retraite à des Jésuites : depuis Saint Ignace, c'était peut-être la première fois que ce n'était pas un jésuite qui faisait la retraite aux Jésuites ! Sans doute, donnait-il l'impression de dire toujours la même chose, mais sans vraiment se répéter. Car son message allait de plus en plus à l'essentiel. Et plus il se rapprochait de cet essentiel, plus son enseignement était neuf. Sa manière de scruter le réel sans jamais l'épuiser s'apparentait de plus en plus à la méthode contemplative des mystiques. Et son public, fidèle, ne se lassait pas de l'entendre, tant rayonnait de lui une aura aux accents prophétiques...

\section{Robert Guelluy et les femmes sages}

Dans sa manière de découvrir l'Évangile, il y avait un côté affectif qui touchait particulièrement son public féminin. « Oh ! Les femmes! Ne m'en parlez pas ! J'ai été l'esclave des femmes pendant toute ma vie ! » Les mouvements féminins, les congrégations religieuses, telle celle du Berlaymont, ne cessaient de faire appel à lui. Il a été « empoisonné » par sa petite « clientèle ». Même celles qui quittaient la vie religieuse continuaient à le poursuivre et à l'écouter. Une douzaine au total. À l'École des sciences philosophiques, une auditrice lui resta fidèle pendant dix-neuf ans ! À ses conférences aux Beaux-Arts, il avait des milliers de femmes comme public. Et sœur Agnès, fondatrice des Annonciades à Louvain-la-Neuve, a eu la naïveté de lui dire un jour - et lui la naïveté de le répéter - que quand elle était derrière 
lui, elle pressait le pas pour le rattraper ; mais que, si elle était devant lui, elle traînait jusqu'à ce qu'il la rejoigne, tant elle était avide de l'entendre. Il ne cachait pas que cette affection était réciproque. Malgré sa très grande réserve, l'émotivité a toujours eu une grande place dans sa vie et c'est elle surtout qui attirait. Il aimait évoquer les « femmes sages » auprès desquelles il avait eu un certain succès : Ginette Carlier, Agnès Coupé et toutes les Coupé, et tant d'autres encore dont il parlait avec un souvenir attendri, parmi lesquelles celles qui l'ont accompagné jusqu'à ses derniers jours...

\section{Jusqu'au bout, toujours libre et frondeur}

Malgré la thrombose qui l'a terrassé en octobre 1999, il resta lucide jusqu'à la fin. Début décembre, dans le home de Néchin, près de Tournai, où il avait accepté de se retirer, il s'en prenait encore, avec ce qui lui restait de vigueur, au culte de la hiérarchie et des dogmes, à l'apparat de la richesse, au souci de la notoriété, à la grandiloquence, au faux sensationnalisme des grands rassemblements de foules, qui pour lui n'avait rien d'évangélique. Son Dieu était le Dieu de la liberté et non le Dieu de l'ordre, le Dieu des pauvres et des petits et non celui des nantis. Le Dieu qui ne cesse de faire des merveilles avec rien. Oui, les hommes ont besoin de symboles, mais de symboles authentiques qui répondent à ce qu'ils vivent. Il continuait à penser que Monseigneur Gaillot n'avait pas dit son dernier mot..., que l'histoire n'était pas finie. 
Le mercredi 26 janvier 2000, son débit était devenu très lent et sa voix peu audible. Mais sa vivacité d'esprit était intacte. Il se remémorait quelques bonnes blagues de sa jeunesse. Notamment celle des deux prélats auxquels le même jour il arriva de tomber par terre, en pleine cérémonie publique, croyant s'asseoir sur un siège qui n'était pas là ! Il avait seize ou dix-sept ans à l'époque. Le premier incident s'était passé à Tournai, lors d'une cérémonie de confirmation. C'était le directeur du collège qui avait retiré la chaise sur laquelle l'évêque croyait s'asseoir. Le second eut lieu à l'université à l'occasion d'une ouverture académique. Là personne n'avait retiré de siège, mais lorsque le prélat est tombé, il a demandé : «Qui a retiré la chaise ?» De ce double événement survenu le même jour il tirait comme leçon que lorsqu'on représente l'autorité on a bien plus de difficulté à vivre des situations burlesques que lorsqu'on est enfant de chœur...

Le problème de l'exercice de l'autorité dans l'Église le préoccupait toujours beaucoup. Il déplorait le centralisme romain. « L’Évêque de Tournai n'a pas plus de pouvoir dans l'Église que moi » disait-il. Il regrettait que le Pasteur Roger Schutz, vu son grand âge, reste accroché à sa fonction à Taizé, empêchant le renouveau que pourraient y insuffler de plus jeunes. Il estimait que ce serait un signe de profond respect de dire à un vieil homme la vérité sur ses défaillances et de l'inviter à se retirer. Il appréciait le fonds authentique des spiritualités orientales mais trouvait excessif l'engouement dont elles sont actuellement l'objet. 
Il n'arrêtait pas d'aborder encore bien d'autres thèmes qui lui tenaient à cœur : le célibat des prêtres, l'avortement, les questions sociales... C'est dire que les derniers mois de sa vie, il était toujours bien ancré dans les problèmes de ce monde. En homme pacifié, mais toujours libre et frondeur, jusqu'au bout il resta attentif aux signes des temps et, dans son franc parler, osait (comme il aimait ce verbe oser !) miser sur l'avenir. S'il était physiquement fort diminué, sa vision de l'existence n'avait pas changé pour autant. Il vivait son état comme un état de grâce. Dans la confiance. « Ma prière constante est une prière de gratitude. Je suis sûr de Dieu. Les saints ne m'intéressent plus. Je regarde directement Jésus-Christ et c'est l'Évangile qui me nourrit. Je ne le lis plus chaque jour, mais je l'écoute avec grande attention le dimanche. Tout est tellement simplifié. Je suis assez fatigué, il est vrai, mais il faut accepter la réalité telle qu'elle est, dans l'abandon. Tout le réel est inconnu. » Ce sont ses derniers mots qui ont été recueillis.

Cet enfant heureux, cet homme heureux, ce professeur heureux, ce prêtre heureux, qui bénissait la chance qu'il avait eue de faire toujours ce qui l'intéressait, s'est éteint en dormant, dans la paix, le mardi 6 février 2001. Sur son souvenir mortuaire est repris le texte de Michée 6.8 : 
On t'a fait savoir, ô homme, ce qui est bien ce que Jahvé réclame de toi :

rien d'autre que d'accomplir la justice, d'aimer la bonté et de marcher avec ton Dieu. 


\section{Robert Guelluy Sa pensée}

La pensée de Robert Guelluy est présentée ici sous la forme de citations extraites de ses œuvres et classées par ordre alphabétique des thèmes. C'est lui-même qui, avant sa mort, a procédé à cette sélection, sur base d'un corpus de 675 citations que j'avais collationnées dans l'ensemble de ses publications. Chacune des références de ces courts textes renvoie à la bibliographie « Robert Guelluy : son œuvre ». 


\section{$A$}

$\underline{\text { Retour à la table des matières }}$

Accueil

L'unique nécessaire, c'est l'accueil, à Dieu et aux autres.

Mais il y a Jésus-Christ, p. 36.

\section{Action}

Notre action sera profonde dans la mesure où notre âme le sera.

À l'écoute de Dieu, p. 162.

\section{Admirer}

La faculté d'admirer est, dans toute âme, égale à sa capacité de s'oublier $[\ldots]$

À l'écoute de Dieu, p. 132.

Affection

Une affection vraie est toujours transformante.

L'affection est éveilleuse d'âme [...]

À l'écoute de Dieu, p. 179.

L'Immaculée Conception, p. 48. 
Toute affection dont on est sûr libère.

Présence de Dieu, p. 24.

[...] l'affection purifie le regard : seul le regard du cœur est véridique.

Si tu connaissais, p. 167.

Agir

Il faut parfois de la force pour ne pas agir.

Faire fleurir l'instant, p. 27.

Aimer

Aimer est une folie, mais en ce bas-monde la folie seule est raisonnable.

À l'écoute de Dieu, p. 201.

[...] on n'aime que dans la joie, on ne connaît la joie que dans l'oubli de soi.

À l'écoute de Dieu, p. 52.

Nous consentons plus volontiers à aider qu'à aimer.

Mais il y a Jésus-Christ, p. 138.

Nul n'est banal pour qui sait l'aimer.

Marie et la présence du Christ en nos vies, p. 13.

[...] on aime toujours au-delà des raisons d'aimer.

Si tu connaissais, p. 132. 
Ame

Nous sommes trop souvent absorbés par nos occupations matérielles pour avoir le temps d'avoir une âme [...]

À l'écoute de Dieu, p. 219.

$A m i$

Il est plus facile de se faire des disciples que des amis !

À l'écoute de Dieu, p. 46.

\section{Amitié}

Une amitié est un luxe, non un placement dont on calcule les rendements $[\ldots]$

De la paternité de Dieu aux pères et mères de ce monde, p. 41.

\section{Amour}

[...] c'est la loi de l'amour qu'on reçoit dans la mesure où l'on donne, qu'on se possède dans la mesure où l'on se perd, qu'on devient soi-même dans la mesure où l'on se renonce.

L'Église, mystère de salut, p. 18.

L'amour modèle celui qui est aimé [...]

L'Immaculée Conception, p. 49. 
[...] l'amour est plus fort que la mort $[\ldots]$

Mais il y a Jésus-Christ, p. 113.

\section{Apparence}

Le réel le plus réel ce que nous sommes le plus profondément est tellement plus que ce qui se voit. Nous sommes tellement plus que nos apparences.

Faire part, p. 61.

\section{Appel}

Aucun de nos moments n'est sans appel divin ni sans grâce pour y répondre.

Mais il y a Jésus-Christ, p. 92.

\section{Argent}

Il est bien difficile de se servir de l'argent sans le servir, or l'argent est un maître dur; il faut plaindre ses esclaves. Pour en user avec une âme libre, il faut être capable de s'en passer ...

À l'écoute de Dieu, p. 201.

Artisan

L'établi où peine l'artisan est aussi le banc d'essai où s'éprouve son âme [...] 


\section{Autrui}

Pour ne pas être un mort vivant, il faut vivre avec autrui et pour autrui.

À l'écoute de Dieu, p. 177.

Estimer autrui, c'est confesser que nous trouvons en lui des valeurs qui nous aident à devenir nous-mêmes.

Pourquoi l'apostolat? , p. 10.

\section{Avancer}

Il faut persévérer : c'est en avançant que chacun identifie mieux la voie qu'il doit suivre.

À l'écoute de Dieu, p. 59.

\section{Avenir}

Ne plus avoir d'avenir, c'est ne plus exister, c'est survivre. Vivre, c'est avoir un avenir, une espérance.

L'Espérance, p. 25.

Nous ne sommes jamais sans avenir $[\ldots]$ 


\section{Avoir}

$[\ldots]$ nous sommes prisonniers de ce que nous avons $[\ldots]$

À l'écoute de Dieu, p. 176.

[...] les malheurs des hommes viennent de ce qu'ils conjuguent trop le verbe avoir et pas assez le verbe être.

Mais il y a Jésus-Christ, p. 87. 


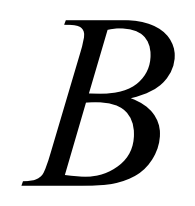

$\underline{\text { Retour à la table des matières }}$

Beau

Si l'homme rend le monde plus beau, c'est qu'il le transforme en miroir de son âme $[\ldots]$

Le Travail dans la vie du chrétien, p. 38.

\section{Bon}

L'homme bon habite un ermitage intérieur peuplé d'affections.

Mais il y a Jésus-Christ, p. 140.

L'homme bon n'a que des proches.

Mais il y a Jésus-Christ, p. 141.

Bonheur

[...] le bonheur est toujours dans une générosité; c'est ce qui le distingue du plaisir, qui attache l'homme à lui-même [...]

À l'écoute de Dieu, p. 31.

[...] un bonheur et une joie valent ce qu'ils coûtent [...]

Faire part, p. 10. 


\section{Bonté}

[...] rien de bon n'est en nous qui ne vienne de la source de toute bonté $[\ldots]$

À l'écoute de Dieu, p. 171. 


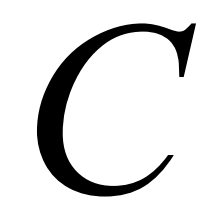

$\underline{\text { Retour à la table des matières }}$

Charité

[...] là où il y a charité vraie jaillissent des miracles.

À l'écoute de Dieu, p. 162.

La charité fait de notre terre l'antichambre des cieux.

Unis dans le peuple de Dieu, p. 23.

Chrétien

Vivre en chrétien, c'est vivre en se sachant aimé.

À l'écoute de Dieu, p. 172.

Ciel

Nous devons vivre ici-bas en citoyens des cieux : vivre vraiment ici-bas, les yeux ouverts sur tous les problèmes qui s'y posent, vivre pourtant en citoyens des cieux, en portant sur toutes choses un autre regard que celui du sage de ce monde.

À l'écoute de Dieu, p. 125. 
Notre vie chrétienne est le ciel commencé; c'est mal comprendre le ciel que d'en oublier la présence anticipée.

À l'écoute de Dieu, p. 97.

$[\ldots]$ le ciel n'est pas étranger à la terre $[\ldots]$ notre éternité commence dans nos fugitives journées.

Le Travail dans la vie du chrétien, p. 73.

Dieu ne nous donne pas le ciel tout fait, il nous le donne à faire [...]

Vie de foi et tâches terrestres, p. 174.

\section{Civilisation}

Faire progresser la civilisation, c'est plus ou moins directement mettre l'homme en mesure d'aller à Dieu avec tout ce que Dieu a mis en lui.

L'Église, mystère de salut, p. 15.

\section{Communication}

[Il faut] se convertir de l'information à la communication. L'information est à sens unique, la communication est faite d'échanges où tous ont à donner en même temps qu'à recevoir.

Mais il y a Jésus-Christ, p. 169.

\section{Communier}

Communier, c'est vivre en unité. 


\section{Confiance}

Là où il y a mutuel amour, il y a mutuelle confiance; or le propre de la confiance est de reposer sur des signes seulement et non sur des justifications.

À l'écoute de Dieu, p. 164.

[...] la confiance est la forme la plus précieuse de l'honneur fait à quelqu'un.

La Théologie face au travail, à ses fruits, à ses exclus, p. 96.

\section{Conquérir}

[...] en conquérant le monde, c'est aussi lui-même que l'homme conquiert.

À l'écoute de Dieu, p. 120.

\section{Conscience}

On peut agir sur une conscience, l'influencer, la transformer, mais on ne peut la donner : on ne peut qu'assister à son éclosion.

Vie de foi et tâches terrestres, p. 25.

$[\ldots]$ la conscience $[\ldots]$ n'a pas d'ancêtres.

Vie de foi et tâches terrestres, p. 26.

\section{Contemplatif}

Il faut des contemplatifs qui renoncent au travail afin que ceux qui travaillent gardent dans leur labeur un peu de l'esprit de contemplation. 
Un contemplatif est un «ravi».

Présence de Dieu, p. 65.

[...] un contemplatif est un homme attentif à toutes les dimensions du réel.

Présence de Dieu, p. 7.

\section{Conversion}

$[\ldots]$ une «conversion», c'est un «retournement».

À l'écoute de Dieu, p. 190.

Nous n'avons pas à être des convertis, mais à être en état de conversion.

Présence de Dieu, p. 56.

\section{Création}

Croire à la création c'est voir sous toutes choses quelqu'un [...]

La Création, p. 55.

Créer

Créer, c'est aimer le premier $[\ldots]$

La Création, p. 55.

Croire

Croire est un don.

Mais il y a Jésus-Christ, p. 12.

Croire en Dieu, pour le chrétien, c'est croire en l'homme [...]

Mais il y a Jésus-Christ, p. 16. 


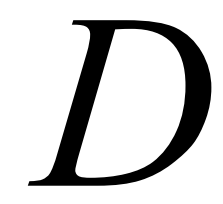

$\underline{\text { Retour à la table des matières }}$

\section{Debout}

Partout où le Christ passe, on se remet debout.

Mais il y a Jésus-Christ, p. 24.

\section{Demain}

[...] demain doit être plus riche qu'hier.

La Théologie face au travail, à ses fruits, à ses exclus, p. 83.

\section{Désespoir}

[...] le désespoir est une des formes de la suffisance.

La Signification chrétienne du travail, p. 18.

\section{Désintéressement}

L'homme n'est jamais aussi vraiment homme que dans le désintéressement.

Vie de foi et tâches terrestres, p. 25.

\section{Destinée}

[...] notre ultime destinée est au-delà de nos forces, au-delà de l'humain. 


\section{Devoir}

L'idéal auquel nous devons tendre n'est nullement de faire par devoir un travail auquel nous refusons de prendre goût, mais d'accomplir avec plaisir le labeur que le devoir nous impose.

Vie de foi et tâches terrestres, p. 115.

\section{Dieu}

Nul n'est si proche de nous que Dieu [...]

À l'écoute de Dieu, p. 27.

[...] Dieu ne nous aime pas parce que nous sommes aimables, mais nous sommes aimables parce que Dieu nous aime.

À l'écoute de Dieu, p. 41.

La véritable image de Dieu, c'est l'homme [...]

À l'écoute de Dieu, p. 68.

Dieu est au bout du chemin et il est aussi tout au long du chemin.

Espérance et fidélité, p. 7.

[...] Dieu seul peut donner Dieu.

L'Église, mystère de salut, p. 28.

[...] nous avons besoin de Dieu plus que de rien au monde.

Le Travail dans la vie du chrétien, p. 153.

[...] Dieu est comme le cœur de notre cœur [...]

Le Travail dans la vie du chrétien, p. 17.

[...] nous mettons Dieu à côté du réel, au lieu de le voir dedans [...] Vie de foi et tâches terrestres, p. 114. 
[...] parce que nous mettons Dieu hors du monde, nous mettons le monde à la place de Dieu.

Vie de foi et tâches terrestres, p. 114.

\section{Différence}

Oser croire à la fécondité des différences.

Faire fleurir l'instant, p. 28.

Ce qui est fécond, c'est la différence. La vie est faite de la conjonction des différences $[\ldots]$

La Gratuité, p. 62.

\section{Dimanche}

[...] il faut un jour qui soit tout entier dimanche pour qu'il y ait un peu de dimanche dans tous les jours de la semaine [...]

Présent et futur de la prière, p. 16.

\section{Discussion}

La discussion a quelque chose de meurtrier, elle vise à réduire l'autre au silence en ayant le dernier mot, c'est-à-dire qu'elle vise à en faire un cadavre. Le silence qui la clôt est un silence fermé, un silence de mort.

La Théologie face au travail, à ses fruits, à ses exclus, p. 66.

\section{Donner}

On peut donner sans être prêt à recevoir et le don est alors sans joie.

À l'écoute de Dieu, p. 213. 


\section{Donner (se)}

On ne se possède que dans la mesure où l'on se donne [...]

Le Travail dans la vie du chrétien, p. 39.

\section{Douceur}

La douceur est signe de force, tandis que la violence trahit la faiblesse, le manque de possession de son âme.

À l'écoute de Dieu, p. 39.

\section{Doux}

Il faut craindre les doux : ils posséderont la terre.

À l'écoute de Dieu, p. 185.

Pour être plus forts, nous devrions être plus doux.

À l'écoute de Dieu, p. 185. 


\section{$E$}

$\underline{\text { Retour à la table des matières }}$

Écouter

La bonté ne se contente pas d'entendre, elle écoute.

Mais il y a Jésus-Christ, p. 139.

\section{Église}

L'Église est, en ce monde qui passe, une présence de l'Éternel [...]

Unis dans le peuple de Dieu, p. 9.

Enfer

[...] l'isolement qu'est l'enfer est le royaume de la vraie mort et des véritables ténèbres.

Vie de foi et tâches terrestres, p. 62.

\section{Ennui}

Au bonheur, il est un obstacle plus grand sans doute que la souffrance : l'ennui, le manque d'élan, le dégoût de la vie, le mécontentement de soi-même et des autres. 
Épreuve

[...] l'épreuve est toujours dans la grâce comme la grâce est toujours dans l'épreuve.

La Gratuité, p. 90.

\section{Espérance}

Nous sommes l'espérance de Dieu.

Mais il y a Jésus-Christ, p. 73.

\section{Espérer}

Espérer, c'est faire face à l'avenir avec une âme neuve [...]

Espérance et fidélité, p. 6.

Nous avons besoin d'espérer pour vivre [...]

Mais il y a Jésus-Christ, p. 22.

\section{Essentiel}

[...] nous compliquons notre vie en l'encombrant de regrets ou d'illusions, nous cherchons loin l'accessoire, alors que l'essentiel est à notre portée.

L'Amour de Dieu et le nôtre, p. 39.

Éternel

Il y a déjà de l'éternel dans l'actuel [...]

L'Espérance, p. 59.

Éternité

[...] Dieu n'a pas voulu nous donner notre éternité toute faite, il nous la donne à faire. 


\section{[...] l'éternité est notre vraie patrie $[\ldots]$}

Le Travail dans la vie du chrétien, p. 24.

Etre

Le trop à «faire» empêche simplement d'être : le souci d'efficience ne laisse plus le loisir d'exister.

Présence de Dieu, p. 43. 


\section{$F$}

$\underline{\text { Retour à la table des matières }}$

\section{Faiblesse}

Ce qui nous perd, ce ne sont pas tellement nos faiblesses, mais plutôt nos duretés $[\ldots]$

Mais il y a Jésus-Christ, p. 72.

\section{Faute}

On se s'oppose pas à Dieu quand on est content de lui; toute faute est le fruit d'une amertume, d'un dépit.

À l'écoute de Dieu, p. 133.

Ce qui est plus grave que nos fautes, c'est le désespoir qui peut les suivre, c'est qu'elles nous fassent douter du cœur du Père.

La Gratuité, p. 147.

Femme

[...] la femme est vraiment elle-même dans l'affection.

Homme et femme, p. 10.

À la place que la femme tient dans une civilisation se reconnaît le cas qu'on y fait de la personne.

Loisirs et délassements, p. 31. 


\section{Fidélité}

[...] la fidélité est la victoire de la vie sur les tentations de mort qui jalonnent le temps.

Mais il y a Jésus-Christ, p. 106.

\section{Foi}

La foi est une lucide et courageuse naïveté.

Faire part, p. 131.

La foi est [...] une présence, dans le temporel, de l'éternel.

Unis dans le peuple de Dieu, p. 22.

\section{Force}

Ce qui nous est demandé en toutes choses, ce n'est ni la faiblesse ni la violence, c'est la force, qui a pour critère la douceur et pour fondement l'humilité.

L'Église, mystère de salut, p. 18.

La force est le privilège du doux [...] 


\section{$G$}

$\underline{\text { Retour à la table des matières }}$

\section{Générosité}

Nos vies sont vides dans la mesure où elles sont remplies de nousmêmes : une vie pleine est une vie où la générosité a pris toute la place.

À l'écoute de Dieu, p. 217.

La générosité est le secret de la joie [...]

L'Amour de Dieu et le nôtre, p. 10.

\section{Génie}

[...] le génie est une longue patience : il faut laisser mûrir et fructifier tout le passé enfoui dans le présent.

Mais il y a Jésus-Christ, p. 119.

\section{Grâce}

La sympathie de Dieu s'appelle la grâce [...]

Faire part, p. 129.

À chacun, Dieu donne sa grâce selon ce qu'il attend.

Le Travail dans la vie du chrétien, p. 76.

[...] nul de nos moments n'est sans grâce [...]

Présence de Dieu, p. 145. 


\section{Gratis}

Vous recevez gratis, donnez gratis !

Mais il y a Jésus-Christ, p. 17. 


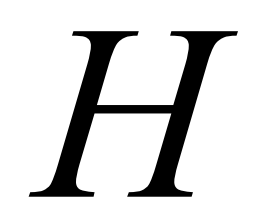

$\underline{\text { Retour à la table des matières }}$

\section{Habiter}

Les lieux que nous habitons nous habitent $[. .$.

Vie de foi et tâches terrestres, p. 55.

\section{Homme}

L'homme vaut $[\ldots]$ ce que vaut son cœur $[\ldots]$

L'Amour de Dieu et le nôtre, p. 10.

[Dieu] a inventé l'homme pour l'établir au-delà de l'humain.

L'Immaculée Conception, p. 44. 
$\underline{\text { Retour à la table des matières }}$

\section{Icône}

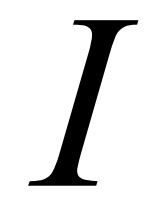

L'icône est aux yeux ce que la parole de Dieu est aux oreilles.

Quelques pensées sur les icônes, inédit.

Toute icône est un appel : elle invite à devenir comme elle, image de Dieu.

Quelques pensées sur les icônes, inédit.

L'icône est l'un des visages que prend la présence de Dieu.

Quelques pensées sur les icônes, inédit.

Une icône naît quand une âme devient, dans la prière, miroir de la lumière divine.

Quelques pensées sur les icônes, inédit.

L'icône est une parole de Dieu, germée dans le silence et fleurie dans la prière.

Quelques pensées sur les icônes, inédit.

L'icône est un programme de vie : elle est un reflet terrestre de la lumière céleste. 
Idée

[...] il nous est difficile de ne pas défendre des idées saines sans nous défendre à travers elles [...]

À l'écoute de Dieu, p. 46.

[...] le réel est bien plus riche que le contenu de nos idées [...]

Présence de Dieu, p. 22.

\section{Instant}

Hier n'est plus, demain n'est pas encore. Seul l'instant présent est à notre disposition.

Prières d'espérance, p. 17.

\section{Invisible}

Les vraies réalités sont invisibles. Ce que nous sommes le plus profondément est ce qui se devine le moins aisément.

Mais il y a Jésus-Christ, p. 112.

[...] il faut se ménager assez de recueillement pour voir l'invisible sous l'immédiatement perceptible.

Mais il y a Jésus-Christ, p. 23.

Quand l'invisible sera rendu visible se révèlera le vrai visage du visible.

Vie de foi et tâches terrestres, p. 56.

\section{Isolé}

Celui qui se suffit n'a besoin ni de Dieu, ni des autres. C'est un isolé. Et être isolé, c'est être mort-vivant. 


\section{Isolement}

\section{$[\ldots]$ l'isolement tue la vie $[\ldots]$}

Unis dans le peuple de Dieu, p. 7. 
Joie

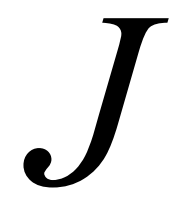

Nul ne trouve en lui-même sa joie $[\ldots]$

À l'écoute de Dieu, p. 212.

La joie rayonne comme un pressentiment de l'éternité.

Faire fleurir l'instant, p. 26.

Il nous est plus difficile d'accueillir la joie que la tristesse; nous sommes spontanément moins portés à douter des mauvaises nouvelles que des bonnes; nous sommes plus disposés à mettre les choses au pis qu'à envisager les prévisions favorables, plus enclins à redouter qu'à espérer, plus frappés par les difficultés que par les promesses de succès, plus impressionnés par les échecs que par les réussites, plus prompts à remarquer les défauts que les qualités ...

Vie de foi et tâches terrestres, p. 15.

\section{Joue gauche}

Tendre la joue gauche n'est pas une capitulation, mais une façon extrême d'acculer ses ennemis à se remettre en question.

Faire part, p. 89.

\section{Juste}

[...] il y a parfois plus de générosité à être juste qu'à être «généreux». 


\section{Justice}

[...] la justice de Dieu, c'est sa justesse : Dieu est juste à la manière dont une note de musique est juste [...] 


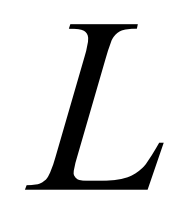

$\underline{\text { Retour à la table des matières }}$

Langage

Le langage est un instrument de la pensée; il n'est pas fait pour la remplacer.

À l'écoute de Dieu, p. 192.

[...] ce monde est un langage de Dieu [...]

Vie de foi et tâches terrestres, p. 113.

Langue

Nous ne pouvons pas comprendre sans nous soumettre à la structure d'une langue. Une grammaire est indispensable pour se rencontrer $[\ldots]$

Mais il y a Jésus-Christ, p. 158.

\section{Liberté}

La liberté n'est que la spontanéité de l'amour; être libre, c'est pouvoir aimer.

Aujourd'hui comme toujours l'homme ne vit pas seulement de pain, p. 7.

[...] la liberté humaine doit être libérée [...]

Liberté et gratuité, p. 140. 


\section{Libre}

[...] nos actes les plus libres [...] ne sont pas ceux que nous avons le plus raisonnés, ceux qui ont été précédés de plus de calculs, mais ceux qui sont posés dans une plus grande unité d'âme, dans plus de recueillement.

Aujourd'hui comme toujours l'homme ne vit pas seulement de pain, p. 9.

Seuls les hommes heureux sont libres. L'homme malheureux est prisonnier de sa tristesse et de son défaitisme.

L'Espérance, p. 48.

\section{Loisir}

Pour bien user des loisirs, il faut un cœur sans égoïsme. C'est une fort grosse naïveté de prétendre diviser sa vie en deux parts : celle où l'on fait ce qu'on doit et celle où l'on fait ce qu'on veut; le devoir est présent partout et c'est partout qu'il faut avoir une âme libre.

À l'écoute de Dieu, p. 202.

Le loisir n'est pas [...] un luxe superflu, mais une condition indispensable de triomphe de l'esprit dans les créatures matérielles que nous sommes; tout homme a besoin, pour vivre en être libre, d'un minimum de temps dont il puisse disposer librement.

Loisirs et délassements, p. 12. 


\section{$M$}

$\underline{\text { Retour à la table des matières }}$

\section{Machine}

[...] aucune machine n'est innocente; chacune façonne celui qui l'utilise : nos outils nous transforment, nos maisons nous habitent.

Présent et futur de la prière, p. 9.

\section{Mariage}

[...] le mariage n'est pas une organisation de bienfaisance : on n'épouse pas quelqu'un pour faire son bonheur; on épouse quelqu'un pour recevoir de lui son bonheur, dans un partage.

Valeurs de la jeunesse d'aujourd'hui, p. 7.

[...] un mariage d'amour n'est pas un mariage de raison, et cependant ce qu'il comporte de folie peut et doit être raisonnable ...

Vie de foi et tâches terrestres, p. 146.

\section{Mépris}

Rien ne blesse un individu ou une communauté comme la mésestime, le sentiment d'être incompris ou exploité. Le mépris est plus lourd à porter que la misère; les pauvres ont moins faim de pain que d'amis $[\ldots]$

Espérance et fidélité, p. 12.

[...] il y a des paroles qui tuent, aussi sûrement qu'un coup de feu. Un coup de feu tue de mort subite, une parole de mépris tue au terme d'une longue agonie. 


\section{Meurtre}

[...] là où il y a mise à mort, il $\mathrm{y}$ a corrélativement suicide : on n'attente pas à la vie d'autrui sans détruire sa propre vie.

La Théologie face au travail, à ses fruits, à ses exclus, p. 66.

\section{Miséricorde}

Dieu n'est jamais aussi entièrement lui-même que dans la miséricorde.

Mais il y a Jésus-Christ, p. 79.

\section{Moi}

Ce qui nous ruine, physiquement et spirituellement, c'est de penser à nous-mêmes : le moi est la plus harassante des compagnies.

À l'écoute de Dieu, p. 202.

\section{Morale}

Il n'y a finalement, à la base de toute morale, qu'un seul précepte : devenir ce qu'on est.

L'Esprit communautaire surnaturel, p. 5.

\section{Mort}

Notre mort est commencée : secrètement elle nous habite.

À l'écoute de Dieu, p. 179.

[...] la mort chrétienne n'est pas la rupture des liens qui nous unissent, elle est le commencement d'une union où nos proches nous deviendront indiciblement proches et où les étrangers seront, avec une authenticité sans réticence, nos frères. 
Nous savons que nous allons vers la Vie, mais que la mort est la porte d'entrée.

Mais il y a Jésus-Christ, p. 31.

\section{Mortification}

On a trop souvent parlé de mortification, alors qu'il faudrait, pour rester en concordance avec l'Évangile, parler de «vivification».

Mais il y a Jésus-Christ, p. 91.

Les mortifications volontaires n'ont pas de valeur en elles-mêmes, mais comme signes d'espérance en un au-delà de nos satisfactions immédiates.

Si tu connaissais, p. 137.

Seul l'amour permet de vouloir la mortification d'une façon qui ne soit pas morbide. Si le sacrifice était le but, au lieu d'être seulement un moyen d'aimer, si la mort à soi-même n'était au service de l'affection, tout ce renoncement serait indéfendable.

Vie de foi et tâches terrestres, p. 146.

\section{Mot}

Les mots ne sont pas seulement le vêtement du message, ils sont les matrices de la pensée.

Mais il y a Jésus-Christ, p. 172.

\section{Mourir}

$[\ldots]$ c'est $[\ldots]$ progressivement mourir que vivre d'une vie mortelle dont chaque heure hâte la fin.

À l'écoute de Dieu, p. 179.

\section{Mystique}

Les mystiques ne sont pas des rêveurs, mais des voyants [...] 


\section{Offrir}

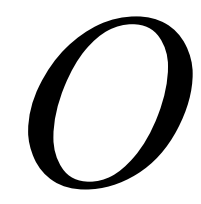

Il n'y a pas, dans la vie chrétienne, de déchets : tout y est richesse, puisque tout peut être offert.

À l'écoute de Dieu, p. 183.

\section{Orgueil}

[...] l'orgueil, c'est d'être occupé de soi et l'humilité, c'est de ne plus penser à soi-même [...] L'humilité nous échappe constamment dans la mesure où nous voulons la cultiver; nous tâchons, par orgueil encore, de devenir humbles!

Croire, c'est vivre en se sachant aimé, p. 11.

L'orgueil est partout une menace pour l'amour.

Mais il y a Jésus-Christ, p. 149.

[...] s'établir dans l'orgueil n'est pas choisir la liberté mais sa caricature.

Vie de foi et tâches terrestres, p. 61.

\section{Oser}

Chaque être humain est une nouveauté, dont Dieu attend du neuf. Sa vocation est d'oser. 


\section{Oui}

Le ciel est la patrie du oui, l'enfer le lieu où l'on est fixé dans le non.

À l'écoute de Dieu, p. 193.

Le «oui» est élevé à la dignité de parole sacramentelle dans le mariage.

À l'écoute de Dieu, p. 193.

Le sommet de la grandeur humaine n'est pas dans le «non» mais dans le «oui».

Aujourd'hui comme toujours l'homme ne vit pas seulement de pain, p. 9.

Le petit enfant dit «non» longtemps avant de dire «oui»; [...] il commence aussi par parler de lui-même à la troisième personne [...] L'aptitude à dire «oui» et la capacité de dire «je» sont solidaires, elles dépendent de l'avènement de l'esprit.

Vie de foi et tâches terrestres, p. 25. 


\section{$P$}

$\underline{\text { Retour à la table des matières }}$

\section{Paix}

[...] notre paix ne nous vient pas de ce que nous sommes sûrs de nous-mêmes, mais sûrs du cœur de Dieu.

L'Amour de Dieu et le nôtre, p. 7.

\section{Pardonner}

[...] pardonner est plus difficile que de ressusciter les morts [...]

Mais il y a Jésus-Christ, p. 81.

\section{Parfaire}

Nous sommes des êtres à parfaire placés dans un monde à parfaire $[\ldots]$

Le Travail dans la vie du chrétien, p. 44.

\section{Partage}

[...] nous devons nous convertir de l'aumône au partage.

Mais il y a Jésus-Christ, p. 170.

\section{Passé}

[...] le passé, sans cesse dépassé, invite à construire un avenir qui le prolonge et l'épanouisse.

Faire part, p. 94. 
[...] le passé fait inventer de l'avenir ...

Mais il y a Jésus-Christ, p. 119.

\section{Pauvre}

Devenir pauvre est une épreuve plus grande que de continuer à l'être; devenir riche menace l'équilibre de la personnalité plus que de le rester.

À l'écoute de Dieu, p. 48.

Nous devons user des richesses avec une âme de pauvre et être pauvre avec une âme d'homme comblé.

À l'écoute de Dieu, p. 156.

Péché

[...] il n'est qu'un seul péché, et c'est la solitude consentie.

L'Église, mystère de salut, p. 20.

$[\ldots]$ la superbe $[\ldots]$ est la sève de tout péché.

Vie de foi et tâches terrestres, p. 61.

Pécher

[...] ma capacité de pécher n'arrivera jamais à bout de la capacité divine de pardonner.

La Gratuité, p. 58.

\section{Perdre (se)}

Il faut se perdre pour se trouver $[\ldots]$

À l'écoute de Dieu, p. 198.

Père de famille

Le métier de père de famille est [...] une carrière d'aventurier.

Présence de Dieu, p. 26. 


\section{Plaisir}

[...] la poursuite du plaisir ferme l'âme, tandis que l'oubli de soi l'épanouit.

À l'écoute de Dieu, p. 32.

Dans le plaisir, [l'homme] s'échappe à lui-même; il ne se possède vraiment que dans le bonheur, qui est toujours le fruit de la générosité.

Aujourd'hui comme toujours l'homme ne vit pas seulement de pain, p. 7.

\section{Présent}

Nous vivons d'ordinaire au passé ou au futur : nous regrettons ce qui n'est plus, nous rêvons de ce qui sera... peut-être, ou nous le redoutons. Or, le passé n'est plus, l'avenir n'est pas encore; l'instant présent seul est à notre disposition.

À l'écoute de Dieu, p. 194.

\section{Prévoyant}

[...] à vouloir être sagement prévoyant, [...] on est fort exposé à devenir égoïste.

Vie de foi et tâches terrestres, p. 127.

\section{Prier}

[...] savoir prier, c'est avant tout savoir se taire en d'autres termes savoir écouter parce qu'on est sûr que Dieu veut faire entendre sa parole.

À l'écoute de Dieu, p. 54.

[...] c'est souvent la difficulté de prier qui évolue en difficulté de croire.

Mais il y a Jésus-Christ, p. 128.

Prier, ce n'est pas d'abord parler, c'est d'abord écouter. 
Prendre conscience devant Dieu des imperfections de la prière, de l'incapacité de prier, [...] c'est encore prier.

Présence de Dieu, p. 56.

Nul ne peut prier, sinon Dieu lui-même dont les pensées deviennent en nous nos pensées.

Présence de Dieu, p. 56.

Prier, c'est espérer.

Présent et futur de la prière, p. 16.

Prière

Être un homme de prière, c'est être un homme qui a lucidement plus ou moins lucidement l'audace d'aller de l'avant en avouant ses limites et en faisant confiance au «maitre de l'impossible».

Présence de Dieu, p. 56.

\section{Psychiatre}

Ce qui annihile l'homme et fait la clientèle des psychiatres, c'est le tête-à-tête avec soi [...]

Vie de foi et tâches terrestres, p. 71. 


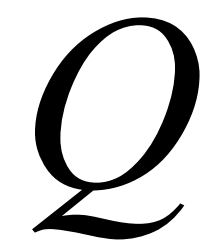

$\underline{\text { Retour à la table des matières }}$

\section{Quelqu'un}

\section{[...] être quelqu'un au lieu d'être quelconque [...]}

Vie de foi et tâches terrestres, p. 195. 


\section{$R$}

$\underline{\text { Retour à la table des matières }}$

Recevoir

Il ne faut pas moins de générosité pour recevoir que pour donner $[\ldots]$

À l'écoute de Dieu, p. 32.

Nous sommes créés : être, pour nous, c'est nous recevoir.

L'Amour de Dieu et le nôtre, p. 5.

Reconnaissance

[...] la reconnaissance est la fleur la plus délicate de la charité.

À l'écoute de Dieu, p. 37.

Recueillement

Le recueillement d'une âme est à la mesure de son détachement $[\ldots]$

À l'éconte de Dieu, p. 184.

Recueilli

Être recueilli, c'est être capable d'écouter; ce n'est pas seulement être capable de vivre seul avec ses propres pensées!

Présence de Dieu, p. 46. 


\section{Réel}

Le vrai réel est au-delà du perçu.

Vie de foi et tâches terrestres, p. 63.

\section{Relation}

Nous valons ce que valent nos relations $[\ldots]$

L'Espérance, p. 19.

\section{Repentir}

Le remords est un face à face avec soi-même, il est attristant et inhibant. Le repentir est l'émotion de retrouvailles, il est heureux et libérant.

Mais il y a Jésus-Christ, p. 60.

Tandis que la présence à soi-même dans les remords est stérile, la présence à Dieu dans le repentir est féconde. D'un côté c'est l'isolement, de l'autre c'est la communion [...]

Vie de foi et tâches terrestres, p. 71.

\section{Repliement}

[...] le pire ennemi de l'homme c'est le repliement sur soi, sous les diverses formes qu'il revêt et qui sont toujours une paralysie ou une négation de l'amour.

L'Amour de Dieu et le nôtre, p. 10.

\section{Respect}

Nous avons plus besoin de respect et d'affection que de nourriture. 


\section{Respiration}

La respiration, c'est la vie, qui vient du plus profond du corps.

Mais il y a Jésus-Christ, p. 147.

\section{Réussir}

Autre chose est de réussir sa vie ou de réussir dans la vie, comme autre chose est le savoir et autre chose le savoir-faire. On peut accumuler les réussites ou le savoir sans parvenir au bonheur.

Si tu connaissais, p. 167.

\section{Richesse}

Les richesses ne sont en elles-mêmes ni bonnes ni mauvaises : elles sont un bien ou un mal selon l'usage que nous en faisons.

Les dons gratuits de Dieu sont nos seules vraies richesses.

À l'écoute de Dieu, p. 176.

Faire fleurir l'instant, p. 43.

\section{Risquer}

Nous avons tous à prendre des risques. Vivre, c'est aimer, aimer c'est risquer, du risque de l'espérance.

La Théologie face au travail, à ses fruits, à ses exclus, p. 95. 


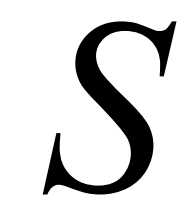

$\underline{\text { Retour à la table des matières }}$

\section{Sage}

Pour être sage, il faut être un peu fou.

À l'écoute de Dieu, p. 218.

\section{Satan}

Satan, c'est en nous le sceptique ou le désabusé qui refusent de faire confiance à la vie et à l'homme : c'est l'être amer que nous sommes souvent, qui, sous prétexte de voir les choses telles qu'elles sont, s'empoisonne l'âme de défiance.

À l'écoute de Dieu, p. 170.

Satan, c'est nous-mêmes qui, en prétendant nous défendre de toute illusion, nous rendons incapables d'espérance et d'amour.

À l'écoute de Dieu, p. 170.

\section{Savoir}

Celui qui pense savoir cesse d'être à même d'apprendre.

À l'écoute de Dieu, p. 14.

\section{Semence}

[...] l'arbre est tout entier dans la semence et pourtant il y a plus dans le chêne que dans le gland. 


\section{Sens}

[...] un monde dont Dieu est absent sera de plus en plus hostile à l'homme et de plus en plus vide de sens.

À l'écoute de Dieu, p. 118.

Nous avons profondément besoin que nos vies aient du sens.

Faire part, p. 121. sens.

[...] nos vies, si misérables qu'elles soient, ne sont jamais vides de Le Travail dans la vie du chrétien, p. 76.

\section{Silence} $[\ldots]$

[...] le silence et la parole se fournissent mutuellement leur sève La Création, p. 101.

[...] la parole dépend normalement de la qualité du silence dont elle jaillit.

Présence de Dieu, p. 76.

\section{Souffrance}

Seul celui qui a souffert sait ce qu'est la souffrance...

À l'écoute de Dieu, p. 11.

Toute souffrance est une invitation à un approfondissement d'âme.

À l'écoute de Dieu, p. 33.

[...] une souffrance qui n'est plus solitaire a changé déjà de visage, elle est tellement autre, elle cesse tellement d'être seulement une souffrance, elle reste une souffrance, mais elle est une forme d'amour. 
[...] il n'y a pas d'existence humaine qui ne soit, un jour ou l'autre, visitée par une grande souffrance.

Mais il y a Jésus-Christ, p. 21.

Les réussites spirituelles valent ce qu'elles coûtent; la souffrance exige un effort qu'on n'aurait pas eu, sans elle, la force de s'imposer. Bien des âmes de qualité lui doivent leur ascension. On peut même dire qu'elle est irremplaçable et qu'on reconnait presque toujours ceux qu'elle a épurés.

Vie de foi et tâches terrestres, p. 172.

\section{Superflu}

[...] l'âme s'embourbe d'une façon plus perverse dans le superflu que dans le manque du nécessaire.

Loisirs et délassements, p. 22.

\section{Surnaturel}

L'ordre surnaturel n'est pas surajouté à l'ordre naturel : il en est la raison d'être.

À l'écoute de Dieu, p. 126.

\section{Sympathie}

[...] les sympathies que nous rencontrons nous raniment comme la rosée fait qu'une plante en train de se flétrir se redresse. 


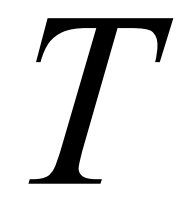

$\underline{\text { Retour à la table des matières }}$

Terre promise

La terre, c'est la terre promise $[\ldots]$

Mais il y a Jésus-Christ, p. 144.

Tolérance

Il n'est qu'une forme vraie de la tolérance et c'est l'affection.

Pourquoi l'apostolat? , p. 15.

\section{Tolérant}

La tolérance n'est souvent que le luxe du plus fort [...]

Mais il y a Jésus-Christ, p. 191.

Être chrétiennement tolérant, c'est aimer tous ceux que Dieu aime $[\ldots]$

L'Esprit communautaire surnaturel, p. 39.

\section{Tragique}

Nos vies ont toutes quelque chose de tragique, ne fût-ce que par leur brièveté. 


\section{Travail}

Le travail n'est pas seulement la transformation du monde par l'homme, il est un façonnement de l'humanité elle-même.

La Création, p. 100.

Exclure quelqu'un du monde du travail, ce n'est pas seulement le priver de moyens de vivre, mais d'une façon humaine de vivre. C'est lui interdire une façon essentielle de partager l'échange entre humains, la présence à ses semblables, la collaboration au devenir des personnes et des cultures.

La Théologie face au travail, à ses fruits, à ses exclus, p. 70.

[...] la personne humaine a droit au travail et à un travail qui ait du sens.

La Théologie face au travail, à ses fruits, à ses exclus, p. 70.

Le premier bénéfice que nous tirons de notre travail est d'être plus homme $[\ldots]$

Le Travail dans la vie du chrétien, p. 19.

Le monde matériel [...] est un temple où l'homme officie; le travail a quelque chose d'une liturgie, le progrès matériel est une manière d'aller à Dieu.

Vie de foi et tâches terrestres, p. 37.

\section{Travailler}

[...] travailler, c'est se faire en faisant le monde.

La Signification chrétienne du travail, p. 12.

Travailler c'est devenir, travailler c'est progresser [...]

La Signification chrétienne du travail, p. 14. 
Le travailleur lui-même est le premier objet que son travail façonne.

Le Travail dans la vie du chrétien, p. 40.

\section{Travailleur}

Trop souvent, le travail nous fait oublier le travailleur, dans lequel nous ne voyons plus que l'auteur d'un rendement : nous jaugeons le résultat utile d'un labeur en perdant de vue ce qu'un homme y a mis de tout lui-même.

Le Travail dans la vie du chrétien, p. 130.

\section{Tristesse}

Les plus tristes tristesses sont celles que nourrit le retour sur soi.

À l'écoute de Dieu, p. 212. 


\section{$U$}

$\underline{\text { Retour à la table des matières }}$

\section{Utile}

Ce qui nous est le plus nécessaire est d'échapper à l'obsession du plus utile.

À l'écoute de Dieu, p. 198. 


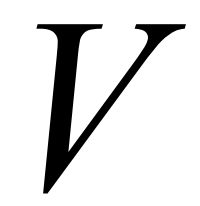

$\underline{\text { Retour à la table des matières }}$

Vie

La vie ne se vit qu'au détail; c'est l'harmonie des détails qui en fait l'homogénéité et nul détail n'est innocent.

Mais il y a Jésus-Christ, p. 169.

Notre vie nous est donnée à inventer; nous connaissons le sens à lui donner, mais il faut dans notre cheminement tracer nos sentiers.

Vie de foi et tâches terrestres, p. 95.

\section{Violence}

La violence ne dure pas, parce qu'elle est l'arme des faibles; ils y brûleront, en peu de temps, toutes leurs ressources.

À l'écoute de Dieu, p. 185.

Virginité

La virginité est une forme de confiance absolue en Celui dont on tient tout et dont on attend tout.

Homme et femme, p. 6.

Visage

Il n'existe pas deux visages humains identiques [...]

Présence de Dieu, p. 58. 
Vivre

[...] c'est bien ça vivre : être toujours conduit là où on ne pensait pas aller.

L'Espérance, p. 21.

Vivre, c'est rayonner.

Présence de Dieu, p. 83.

[...] vivre vraiment c'est aimer, aimer c'est risquer, risquer c'est oser.

Si tu connaissais, p. 136.

Vrai

[...] la fidélité au vrai exige de s'interroger sur le sens caché de la vie, sur la présence de l'invisible dans le visible.

À l'écoute de Dieu, p. 16. 


\section{Robert Guelluy \\ Son œuvre \\ Bibliographie}

$\underline{\text { Retour à la table des matières }}$

Ne sont pas recensés ici les articles courts parus dans diverses revues ni ceux plus importants qui ont été, par la suite, repris comme chapitres de livres. La présente bibliographie peut cependant être considérée comme quasi exhaustive. Elle n'avait jamais été publiée jusqu'ici.

L'Évolution des méthodes théologiques à Louvain, d'Érasme à Jansénius, dans Revue d'histoire ecclésiastique, t. 37, Université Catholique de Louvain, Louvain, 1941, pp. 31-144.

La Place des théologiens dans l'Église et la société médiévales, dans Miscellanea historica in honorem Alberti De Meyer, Recueil de travaux d'histoire et de philologie, $3^{\mathrm{e}}$ série, fasc. 22 , t. 1, Université de Louvain, Louvain/Bruxelles, 1946, pp. 571-589.

Valeur de la prière, dans Le Diocèse de Tournai sous l'occupation allemande, Casterman, Tournai/Paris, 1946, pp. 39-44.

Philosophie et théologie chez Guillaume d'Ockham, Universitas Catholica Lovaniensis, Dissertationes ad gradum magistri in Facultate Theologica vel in Facultate Iuris Canonici consequendum conscriptae, Series II - Tomus 39, Nauwelaerts/Vrin, Louvain/Paris, 1947, 388 p. 
La Distinction des sciences dans le Prologue du Commentaire des Sentences d'Ockham, J. Duculot, Gembloux, 1947. [Cette publication est la reproduction du chapitre VI de la publication précédente, pp. 259-311]

Notes doctrinales, dans La Charité, Campagne Jiciste, Éditions Jicistes, Bruxelles, [1951], $96 \mathrm{p}$.

Notes doctrinales, dans La Mission de la femme, Campagne Jiciste, Éditions Jicistes, Bruxelles, [1953], 96 p.

La Portée religieuse du dogme de la création, dans Nouvelle revue théologique, t. 75, Casterman, Tournai/Louvain, 1953, pp. 803-814.

Le Travail dans la vie du chrétien, Action catholique rurale/J. Duculot, Bruxelles/Gembloux, 1953, 192 p. Paru aussi en italien.

L'Immaculée Conception, J. Duculot, Gembloux, 1954, 63 p.

Marie dans le dessein de Dieu, dans Actes du congrès marial, [Bruxelles], septembre 1954, pp. 27-36. Paru aussi dans Collection Appels, $8^{\mathrm{e}}$ série, $\mathrm{n}^{\circ}$ 6, [1955], pp. 1-9.

L'Église, mystère de salut, dans Les Laïcs dans la crise du monde moderne : responsabilité et formation, Congrès National pour l'Apostolat des Laïcs, Louvain, 29-31 décembre 1956, Éditions de l'Action catholique des hommes, Bruxelles, 1957, pp. 5-34.

L'Esprit communautaire surnaturel, La pensée catholique/Office général du livre, Bruxelles/Paris, 2e éd., 1958, 56 p., collection « Études religieuses $», \mathrm{n}^{\circ} 737$.

Vie de l'Église et intelligence du mystère du salut, dans Lumen Vitae, Revue internationale de la formation religieuse, $t .14, \mathrm{n}^{\circ} 2$, Centre international d'études de la formation religieuse, Bruxelles, 1959, pp. 277-288.

Vie de foi et tâches terrestres, Préface de S. Exc. Mgr [Gabriel-Marie] Garrone, archevêque de Toulouse, Casterman, Tournai, 1960, 203 p., Cahiers de l'actualité religieuse. Paru en néerlandais sous le titre Geloofsleven en aardse opdracht, trad. J. Sterk, De Vroente, Kasterlee, 
1963, 223 p. Paru en anglais sous le titre Christian Commitment to God and to the world, trad. M. Angeline Bouchard, Desclee Company, New York/Tournai/Paris/Rome, 1965, 178 p.

À l'écoute de Dieu, Préface de S. Exc. Mgr [Charles-Marie] Himmer, évêque de Tournai, Casterman, Tournai, 1961, 223 p. Paru en néerlandais sous le titre Luisteren naar God, trad. J. Sterk, De Vroente, Kasterlee, 1962, 208 p. Paru en italien sous le titre In ascolto di Dio, trad. Suore del Carmelo di Leyni, [Torino], Marietti, [1963], 228 p. Paru en anglais sous le titre Tune in to God, trad. Michael C. O'Brien, Desclée Company, New York/Tournai/Rome/Paris, 1968, 268 p.

L'Abbé Jacques Buisseret (en collaboration avec Jacques Leclercq), Éditions Universitaires, Paris/Bruxelles, 1963, 111 p.

La Création, Desclée, Tournai, 1963, 164 p., collection « Le mystère chrétien, section de Théologie dogmatique ». Paru en espagnol sous le titre La Creación, trad. Ruiz Bueno, Herder, Barcelone, 1967, 222 p. Paru en italien sous le titre La Creazione, trad. C. Castiglioni, Desclée, Rome, 1968, 156 p., collection « Il misterio cristiano ». Paru en polonais sous le titre Tajemnica Boga, trad. KS BP Bodhan Bejze Poznan, Ksicgarnia Wojciecha, Varsovie, Lublin, 1966, 456 p., collection « Studia Institute Thomistici », t. 2.

Quel christianisme présenter à la jeunesse ?, dans Lumen Vitae, Revue internationale de la formation religieuse, t. $18, \mathrm{n}^{\circ} 4$, Centre international d'études de la formation religieuse, Bruxelles, 1963, pp. 691-704.

Vivre en Église la sainteté qui nous vient de la Parole et des Sacrements, dans Assemblées du Seigneur, Quinzième dimanche après la Pentecôte, $\mathrm{n}^{\circ}$ 69, Publications de Saint-André, Biblica, [Bruges], [1964], pp. 82-94.

Le Christ aujourd'hui, Éditions de l'Iris, Montréal, 1965, 23 p., collection «Pensées chrétiennes pour le $20^{\mathrm{e}}$ siècle », $\mathrm{n}^{\circ} 1$. 
Marie et la présence du Christ en nos vies, Éditions de l'Iris, Montréal, 1965, 23 p., collection «Pensées chrétiennes pour le $20^{\mathrm{e}}$ siècle $», \mathrm{n}^{\circ} 2$.

Aujourd'hui comme toujours l'homme ne vit pas seulement de pain, Éditions de l'Iris, Montréal, 1965, 15 p., collection « Pensées chrétiennes pour le $20^{\mathrm{e}}$ siècle $», \mathrm{n}^{\circ} 3$.

Aimer, c'est mourir et vivre, Éditions de l'Iris, Montréal, 1965, 15 p., collection « Pensées chrétiennes pour le $20^{\mathrm{e}}$ siècle $», \mathrm{n}^{\circ} 4$.

Croire, c'est vivre en se sachant aimé, Éditions de l'Iris, Montréal, 1965,16 p., collection « Pensées chrétiennes pour le $20^{\mathrm{e}}$ siècle », $\mathrm{n}^{\circ} 5$.

Espérance et fidélité, Éditions de l'Iris, Montréal, 1965, 19 p., collection «Pensées chrétiennes pour le $20^{\mathrm{e}}$ siècle », $\mathrm{n}^{\circ} 6$.

L'Amour de Dieu et le nôtre, Éditions de l'Iris, Montréal, 1965, 44 p., collection « Pensées chrétiennes pour le $20^{\mathrm{e}}$ siècle », $\mathrm{n}^{\circ} 7$.

Unis dans le peuple de Dieu, Éditions de l'Iris, Montréal, 1965, 28 p., collection « Pensées chrétiennes pour le $20^{\mathrm{e}}$ siècle », $\mathrm{n}^{\circ} 8$.

Pécheurs pardonnés, Éditions de l'Iris, Montréal, 1965, 20 p., collection «Pensées chrétiennes pour le $20^{\mathrm{e}}$ siècle », $\mathrm{n}^{\circ} 9$.

Homme et femme, Éditions de l'Iris, Montréal, 1965, 16 p., collection « Pensées chrétiennes pour le $20^{\mathrm{e}}$ siècle », $\mathrm{n}^{\circ} 10$.

De la paternité de Dieu aux pères et mères de ce monde, Éditions de l'Iris, Montréal, 1965, 24 p., collection « Pensées chrétiennes pour le $20^{\mathrm{e}}$ siècle $», \mathrm{n}^{\circ} 11$.

Valeurs de la jeunesse d'aujourd'hui, Éditions de l'Iris, Montréal, 1965, 23 p., collection « Pensées chrétiennes pour le $20^{\mathrm{e}}$ siècle », $\mathrm{n}^{\circ}$ 12. 
La Signification chrétienne du travail, Éditions de l'Iris, Montréal, 1965, 23 p., collection « Pensées chrétiennes pour le $20^{\mathrm{e}}$ siècle », $\mathrm{n}^{\circ}$ 13.

Loisirs et délassements, Éditions de l'Iris, Montréal, 1965, 33 p., collection « Pensées chrétiennes pour le $20^{\circ}$ siècle », $\mathrm{n}^{\circ} 14$.

Pourquoi l'apostolat?, Éditions de l'Iris, Montréal, 1965, 19 p., collection « Pensées chrétiennes pour le $20^{\circ}$ siècle », $\mathrm{n}^{\circ} 15$.

Présence de Dieu, dimensions actuelles de la contemplation chrétienne, Suggestions pratiques pour la prière - Textes pour la soutenir, Casterman, 1970, 195 p., collection « Vivre et croire ». Paru en italien sous le titre Presenza di Dio, trad. E. Galbarati, Cittadellá, Assise, 1970, $182 \mathrm{p}$.

Vivre le défi de Dieu, retraite, Mesvin, été 1974, 95 p.

Le Magistère " ordinaire » chez Paul VI, dans Revue théologique de Louvain, t.9, Louvain-la-Neuve, 1978, pp. 407-416.

De l'héroïsme à la sainteté, dans La Vie des communautés religieuses, t. 37, Montréal, juin 1979, n 6, pp. 184-192.

Les Archives Vincent Lebbe à l'U.C.L., dans Revue théologique de Louvain, t. 12, fasc. 3, 1981, pp. 401-403.

Comment la théologie parle du corps, dans La Signification du corps, Groupe de synthèse de Louvain, Série interdisciplinaire publiée avec le concours de l'ILV de l'UCL, Cabay, Louvain-la-Neuve, 1981, pp. 145-167.

Envoyés pour rassembler, dans Les Paroisses dans l'Église d'aujourd'hui, Louvain-la-Neuve, 14-16 septembre 1981, 16 p.

L'Espérance, Retraite, Uccle, 1981, 70 p. 
Les Exigences méthodologiques d'une théologie des signes des temps, dans Revue théologique de Louvain, t. 12, Louvain-la-Neuve, 1981, pp. 415-428.

Présent et futur de la prière, dans La Prière du chrétien, Publications des Facultés universitaires Saint-Louis, Bruxelles, 1981, pp. 118.

Les Activités du Centre V. Lebbe, dans Revue théologique de Louvain, t. 13, fasc. 4, 1982, pp. 452-455.

Le Théologien dans l'histoire et face au message révélé, dans Responsabilité éthique dans les sciences, Groupe de synthèse de Louvain, Série interdisciplinaire publiée avec le concours de l'ILV de l'UCL, Presses universitaires de Louvain-la-Neuve, Louvain-la-Neuve, 1982, pp. 227-238.

Liberté et gratuité, dans Luther aujourd'hui, publié sous la direction de H.R. Boudin et A. Houssiau, Publications de la Faculté de théologie de Louvain-la-Neuve, Cahiers de la Revue théologique de Louvain, $n^{\circ} 11$, Louvain-la-Neuve, 1983, pp. 133-143.

La Théologie face au travail, à ses fruits, à ses exclus, dans Le Chômage des jeunes, défi aux chrétiens, Colloque organisé par la Faculté de théologie de l'UCL, Vie ouvrière, Bruxelles, 1984, pp. 65-83.

La Foi dans le cas que Dieu fait de nous, Herne, octobre 1985, 102 p.

Les Antécédents de l'Encyclique " Humani generis » dans les sanctions romaines de 1942 : Chenu, Charlier, Graguet, dans Revue d'histoire ecclésiastique, t. 81, n 3/4, Université catholique de Louvain, Louvain-la-Neuve/Leuven, 1986, pp. 421-497.

Mais il y a Jésus-Christ, Duculot, Paris/Louvain-la-Neuve, 1989, $197 \mathrm{p}$.

La Permanence, depuis le XVIe siècle, de deux sensibilités religieuses, dans Revue théologique de Louvain, t. 20, 1989, pp. 308-323. 
La Spiritualité du Père Lebbe, dans Revue Théologique de Louvain, t. 21, 1990, pp. 455-471.

Vincent Lebbe (1877-1940), Pauselijke missiewerken, Missio-cahiers, $\mathrm{n}^{\circ}$ 5, Brussel, 1990, pp. 1-18.

Vivre comme si l'Évangile était vrai : Actualité du Père Lebbe, dans La Revue Générale, décembre 1990, n 12, pp. 19-32.

Si tu connaissais, Duculot, Paris/Louvain-la-Neuve, collection « Spiritualité », 1991, 173 p.

La Souffrance. Approches spirituelles, dans Revue théologique de Louvain, t. 22, 1991, pp. 185-201.

Quelques pensées sur les icônes, inédit, Louvain-la-Neuve, 1991.

Faire part, Racine, Bruxelles, 1993, 144 p.

Vérité et Historicité : le point de vue du théologien, dans Le Supplément, Revue d'éthique et théologie morale, $\mathrm{n}^{\circ} 188-189$, Historicité et Vérité, Cerf, Paris, janvier-juin 1994, pp. 53-79.

Prières d'espérance, Claire-Vision, Louvain-la-Neuve, 1994, 34 p., collection « Prières ».

La Gratuité, Éditions Racine / Fidélité, Bruxelles/Namur, 1995, $157 \mathrm{p}$.

L'Église écoute le monde, dans Vivre autrement le troisième âge. [Les nouveaux béguinages], Actes du colloque de Louvain-la-Neuve à l'occasion de l'inauguration du petit béguinage de Lauzelle, Fraternité Paix et Joie et ARC-B[rabant] W[allon], Lasne, 1996, pp. 71-79.

Faire fleurir l'instant, Fidélité, Namur, 1997, 72 p. 
L'auteur a rédigé aussi des Notes doctrinales pour les Plans de travail 1954-1955, 1955-1956 et 1956-1957 de la Fédération des femmes catholiques belges, Secrétariat national, Bruxelles.

Fin du texte 